\title{
ON THE EXISTENCE OF CERTAIN SINGULAR INTEGRALS.
}

\author{
By
}

\author{
A. P. CALDERON and A. ZYGMUND
}

Dedicated to Professor Marcel Riesz, on the occasion of his 65 th birthday

\section{Introduction.}

Let $f(x)$ and $K(x)$ be two functions integrable over the interval $(-\infty,+\infty)$. It is very well known that their composition

$$
\int_{-\infty}^{+\infty} f(t) K(x-t) d t
$$

exists, as an absolutely convergent integral, for almost every $x$. The integral can, however, exist almost everywhere even if $K$ is not absolutely integrable. The most interesting special case is that of $K(x)=1 / x$. Let us set

$$
\tilde{f}(x)=\frac{1}{\pi} \int_{-\infty}^{+\infty} \frac{f(t)}{x-t} d t .
$$

The function $\tilde{f}$ is called the conjugate of $f$ (or the Hilbert transform of $f$ ). It exists for almost every value of $x$ in the Principal Value sense:

$$
\tilde{f}(x)=\lim _{\varepsilon \rightarrow 0} \frac{1}{\pi}\left(\int_{-\infty}^{x-\varepsilon}+\int_{x+\varepsilon}^{\infty}\right) \frac{f(t)}{x-t} d t .
$$

Moreover it is known (See [9] or [7], p. 317) to satisfy the M. Riesz inequality

$$
\left[\int_{-\infty}^{+\infty}|\tilde{f}|^{p} d x\right]^{1 / p} \leq A_{p}\left[\int_{-\infty}^{+\infty}|f|^{p} d x\right]^{1 / p}, \quad 1<p<\infty
$$

where $A_{p}$ depends on $p$ only. There are substitute result for $p=1$ and $p=\infty$. The limit $\tilde{f}$ exists almost everywhere also in the case when $f(t) d t$ is replaced there by $d F(t)$, where $F(t)$ is any function of bounded variation over the whole interval $(-\infty,+\infty)$. (For all this, see e.g. [7], Chapters VII and XI, where also bibliographical references can be found). 
The corresponding problems for functions of several variables have been little investigated, and it is the purpose of this paper to obtain some results in this direction. To indicate the problems we are going to discuss let us consider two classical examples.

Let $f(s, t)$ be a function integrable over the whole plane, and let us consider in the half-space $z>0$ the Newtonian potential $u(x, y, z)$ of the masses with density $f(s, t)$. Thus

$$
u(x, y, z)=\iint f(s, t) \frac{d s d t}{R}, \quad R^{2}=(x-s)^{2}+(y-t)^{2}+z^{2}
$$

the integration being extended over the whole plane. Let us also consider the partial derivatives

$$
u_{z}=-z \iint f(s, t) \frac{d s d t}{R^{3}}, \quad u_{x}=-\iint f(s, t) \frac{x-s}{R^{3}} d s d t
$$

Here $-(4 \pi)^{-1} u_{z}$ is the Poisson integral of $f$, and it is a classical fact that it tends to $f(x, y)$ as $z \rightarrow 0$, at every point $(x, y)$ at which $f$ is the derivative of its indefinite integral. On the other hand, by formally replacing $z$ by 0 in the formula for $u_{x}$ we obtain the singular integral

$$
\iint f(s, t) \frac{x-s}{\left[(x-s)^{2}+(y-t)^{2}\right]^{3 / 2}} d s d t .
$$

It can be written in the form

$$
\iint f(s, t) K(x-s, y-t) d s d t
$$

with

$$
K(x, y)=\frac{x}{\left(x^{2}+y^{2}\right)^{3 / 2}}
$$

It is a simple matter to show that at every point $\left(x_{0}, y_{0}\right)$ at which $f$ is the derivative of its indefinite integral the existence of the integral is equivalent to the existence of $\lim u_{x}$, as the point $(x, y, z)$ approaches $\left(x_{0}, y_{0}, 0\right)$ non-tangentially (and that both expressions have the same value), but neither fact seems to have been established unconditionally. Here again the integral (2) is taken in the principal value sense, which in two dimensions means that first the integral is taken over the exterior of the circle with center $\left(x_{0}, y_{0}\right)$ and radius $\varepsilon$, and then $\varepsilon$ is made to tend to 0 .

Another example, of a somewhat similar nature, arises from considering in the plane the logarithmic potential $u$ of masses with density $f(s, t)$. Hence 


$$
u(x, y)=\iint f(s, t) \log \frac{1}{r} d s d t ; \quad r^{2}=(x-s)^{2}+(y-t)^{2} .
$$

If in order to avoid unnecessary complications we assume that $f$ vanishes in a neighborhood of infinity, then in any finite circle $u$ is the convolution of two integrable functions, and so the integral converges absolutely almost everywhere. The integral obtained by formal differentiation, say with respect to $x$, is

$$
-\iint f(s, t) \frac{x-s}{(x-s)^{2}+(y-t)^{2}} d s d t
$$

and so, as a convolution of two integrable functions, again converges absolutely almost everywhere and represents a function integrable over any finite portion of the plane. Using this fact one proves without difficulty (see [1]) that the integral actually represents $u_{x}$. Thus $u_{x}$ and $u_{y}$ exist almost everywhere.

Let us, however, differentiate the integral (4) formally once more, with respect to $x$ and with respect to $y$. We get the integrals of type (2) with

$$
K(x, y)=\frac{x^{2}-y^{2}}{\left(x^{2}+y^{2}\right)^{2}}, \quad K(x, y)=\frac{2 x y}{\left(x^{2}+y^{2}\right)^{2}},
$$

respectively. These two kernels are not essentially different, since one is obtained from the other through a rotation of the axes by $45^{\circ}$. It may also be of interest to observe that they appear respectively as the real and imaginary parts of

$$
\frac{1}{z^{2}}=\frac{1}{(x+i y)^{2}}
$$

The existence almost everywhere of the integrals (2) in the cases (5) has been established by Lichtenstein for functions $f$ which are continuous (or, slightly more generally, Riemann integrable). This result seems not to have been superseded so far, though the existence almost everywhere of $u_{x x}, u_{y y}, u_{x y}$ together with the relation $u_{x x}+u_{y y}=-2 \pi f$.was established by Lichtenstein [6] (see also [2], [8]) in the much more general case of $f$ quadratically integrable.

The kernels (3) and (5) have one feature in common: they are of the form

$$
g(\varphi) \varrho^{-2}, \quad x=\varrho \cos \varphi, \quad y=\varrho \sin \varphi,
$$

where $g(\varphi)$ is a function of angle $\varphi$ (actually a trigonometric polynomial) whose mean value over $(0,2 \pi)$ is zero. Several examples of kernels of this type could be considered, but we shall now state the problem in a more general form. 
Suppose we have a function $f\left(x_{1}, x_{2}, \ldots x_{n}\right)$ integrable over the whole $n$-dimensional space, and a kernel

$$
K\left(x_{1}, x_{2}, \ldots x_{n}\right)=\varrho^{-n} \Omega\left(\alpha_{1}, \alpha_{2}, \ldots, \alpha_{n}\right),
$$

where $x_{j}=\varrho \cos \alpha_{j}$ for all $j$, and $\alpha_{1}, \alpha_{2}, \ldots, \alpha_{n}$ are the direction angles. What can be said about the existence and the properties of the integral

$$
\tilde{f}\left(x_{1}, x_{2}, \ldots, x_{n}\right)=\int f\left(s_{1}, s_{2}, \ldots, s_{n}\right) K\left(x_{1}-s_{1}, \ldots, x_{n}-s_{n}\right) d s_{1} \ldots d s_{n} \text { ? }
$$

An answer to this problem is our main object here.

This is the plan of the paper.

In Chapter I it will be shown that, if $f \in L^{p}, 1<p<\infty$, then the integral (6) converges, in the metric $L^{p}$, to a function $\tilde{f} \in L^{p}$, provided

a) the mean value of $\Omega$ over the unit sphere is zero,

b) the function $\Omega\left(\alpha_{1}, \alpha_{2}, \ldots, \alpha_{n}\right)$ satisfies a smoothness condition (See Chapter II).

(In the case $p=2$ condition b) can be considerably relaxed).

The function $\tilde{f}$ satisfies the condition analogous to (1). The cases $p=1$ and $p=\infty$ are also investigated.

The main result of Chapter II is that under conditions a) and b) the integral (6) exists almost everywhere not only for $p>1$, but also for $p=1$. The result holds, if $f d s_{1} \ldots d s_{n}$ is replaced by $d \mu$, where $\mu$ is an arbitrary mass distribution with finite total mass. If $f \in L^{p}, p>1$, the partial integrals of the integral $(6)$, that is the integrals over the exterior of the sphere of radius $\varepsilon$ and center $\left(x_{1}, x_{2}, \ldots x_{n}\right)$ are majorized by a function of $L^{p}$, independent of $\varepsilon$.

Chapter III is devoted to some applications of the results previously obtained to the problem of the differentiability of the potential.

Other problems connected with our main topic will be considered in an another paper.

\section{CHAPTER I.}

\section{Mean Convergence of Singular Integrals.}

Let $E^{n}$ be the $n$-dimensional euclidean space. If $P$ and $Q$ are points in $E^{n}$, $(P-Q)$ will denote either the vector going from $Q$ to $P$, or the point whose coordinates are the components of $(P-Q)$. The length of $(P-Q)$ will be denoted by $|P-Q|$, and $\Sigma$ will stand for the surface of the sphere of radius 1 with center at the origin of coordinates, $O$. 
We shall be concerned with kernels of the form

$$
K(P-Q)=|P-Q|^{-n} \Omega\left[(P-Q)|P-Q|^{-1}\right],
$$

where $\Omega(P)$ is a function defined on $\Sigma$ and satisfying the conditions

$$
\int_{\Sigma} \Omega(P) d \sigma=0
$$

and

$$
|\Omega(P)-\Omega(Q)| \leq \omega(|P-Q|),
$$

where $\omega$ is an increasing function such that $\omega(t) \geq t$, and

$$
\int_{0}^{1} \omega(t) \frac{d t}{t}=\int_{\mathbf{1}}^{\infty} \omega\left(\frac{1}{t}\right) d t<{ }_{t}^{1}
$$

More precisely, we shall investigate the convergence of the integral

$$
\tilde{f}_{\lambda}(P)=\int_{E^{n}} K_{\lambda}(P-Q) f(Q) d Q,
$$

where $f(Q)$ is a function of $L^{p}, p \geq 1$ in $E^{n}, d Q$ is the element of volume in $E^{n}$ and

$$
K_{\lambda}(P-Q)=\left\{\begin{array}{l}
K(P-Q) \\
0 \text { otherwise. }
\end{array} \quad \text { if }|P-Q| \geq 1 / \lambda,\right.
$$

Using Hölder's inequality, or the boundedness of $K_{\lambda}$, we see that (2) is absolutely convergent for $1<p<\infty$ and $p=1$ respectively.

We shall begin by proving that in the case when the function $f$ in (2) belongs to $L_{2} ; \tilde{f_{\lambda}}$ converges in the mean of order two as $\lambda \rightarrow \infty$.

Let

$$
K_{\lambda \mu}=\left\{\begin{array}{l}
K(P-O) \\
0 \text { otherwise. }
\end{array} \quad \text { if } \mu \geq|P-O| \geq 1 / \lambda\right.
$$

As we shall see, the Fourier transform of $K_{\lambda \mu}$ converges boundedly as $\mu$ and $\lambda$ tend to infinity successively, and then the desired result will follow easily.

In polar coordinates we have the following expression for the Fourier transform $\hat{K}_{\lambda \mu}$ of $K_{\lambda \mu}$,

$$
\hat{K}_{\lambda \mu}(P)=\int_{E^{n}} K_{\lambda \mu}(Q) e^{i r \varrho \cos \varphi} d Q=\int_{1 / \lambda}^{\mu} \varrho^{-1} d \varrho \int_{\Sigma} \Omega\left(Q^{\prime}\right) e^{i r \varrho \cos \varphi} d \sigma,
$$

1 This implies the convergence of $\int^{\infty} \omega\left(\frac{c}{t}\right) \frac{d t}{t}$ for every $c>0$, a fact we shall use in what follows.

It may be added that the condition $\omega(t) \geq t$ is quite harmless, since we can always replace $\omega(t)$ by $\operatorname{Max}\{\omega(t), t\}$. The case $\omega(t)=t^{\alpha}, \alpha>0$, is, of course, the most important one. 
where $r=|P-O|, \varrho=|Q-O|, Q^{\prime}=(Q-O)|(i-O)|^{-1}$, and $\varphi$ is the angle between the vectors $(P-O)$ and $(Q-O)$. Introducing the variable $s=\varrho r$ we can write

$$
\hat{K}_{\lambda \mu}(P)=\int_{r / \lambda}^{r \mu} \frac{d s}{s} \int_{\Sigma} \Omega\left(Q^{\prime}\right) e^{i s \cos \varphi} d \sigma,
$$

and owing to the fact that

$$
\int_{\Sigma} \Omega\left(Q^{\prime}\right) d \sigma=0
$$

we also have

$$
\begin{aligned}
\hat{K}_{\lambda \mu}(P) & =\int_{r / \lambda}^{r \mu} \frac{d s}{s} \int_{\Sigma} \Omega\left(Q^{\prime}\right)\left[e^{\left.i s \cos \varphi-e^{-s}\right] d \sigma}\right. \\
& =\int_{\Sigma} \Omega\left(Q^{\prime}\right) d \sigma \int_{r i \lambda}^{r \mu} \frac{e^{i s \cos \varphi}-e^{-s}}{s} d s .
\end{aligned}
$$

Now, if $\varphi \neq \frac{\pi}{2}$, the inner integral in the last expression converges as $\lambda$ and $\mu$ tend to infinity, and it is not difficult to verify that it never exceeds $2 \log \frac{c}{|\cos \varphi|}$ in absolute value, where $c>1$ is a constant. But $\Omega\left(Q^{\prime}\right)$ is a bounded function and the integral

$$
\int_{\Sigma} \log \frac{c}{|\cos \varphi|} d \sigma
$$

is finite, and therefore $\hat{K}_{\lambda \mu}$ is bounded and converges, as $\mu$ and $\lambda$ tend to infinity successively. Therefore, if $\hat{K}_{\lambda}$ is the Fourier transform of $K_{\lambda}(P-O) \in L^{2}$, we have

$$
\lim _{\mu \rightarrow \infty} \hat{K}_{\lambda \mu}=\hat{K}_{\lambda}
$$

and $\hat{K}_{\lambda}$ converges boundedly to a function $\hat{K}$ as $\lambda \rightarrow \infty$.

Let now

$$
\tilde{f}_{\lambda \mu}(P)=\int_{E^{n}} K_{\lambda \mu}(P-Q) f(Q) d Q
$$

Then, if $\hat{\tilde{f}}_{\lambda \mu}$ is the Fourier transform of $\tilde{f}_{\lambda \mu}$, we have

$$
\hat{\tilde{f}}_{\lambda \mu}=\hat{K}_{\lambda \mu} \hat{f}
$$

and since $\hat{K}_{\lambda \mu} \rightarrow \hat{K}_{\lambda}$ boundedly as $\mu \rightarrow \infty$, $\hat{\tilde{f}}_{\lambda \mu}$ converges in the mean to $\hat{K}_{\lambda} \hat{f}$. On the other hand, $\tilde{f}_{\lambda \mu}$ converges to $\tilde{f}_{\lambda}$ as $\mu \rightarrow \infty$ and therefore we have

$$
\hat{\tilde{f}}_{\lambda}=\hat{K}_{\lambda} \hat{f} .
$$


Letting now $\lambda$ tend to infinity, $\hat{K}_{\lambda}$ will converge boundedly to $\hat{K}$, and $\hat{\tilde{f}}_{\lambda}$ will converge in the mean to $\hat{K} \hat{f}$. Therefore $\tilde{f}_{\lambda}$ will converge in the mean to the Fourier transform of $\hat{K} \hat{f}$. This completes the argument.

Remark. In the above argument we used the fact that $Q\left(P^{\prime}\right)$ was merely bounded. Actually the only property of $\Omega$ we need (except for (1)) is the uniform boundedness of $\int_{\Sigma}\left|\Omega\left(Q^{\prime}\right)\right| \log \frac{c}{|\cos \varphi|} d \sigma$. This condition is certainly satisfied if $|\Omega| \log ^{+}|\Omega|$ is integrable.

Before we pass to the general case we shall prove some lemmas which will be needed also in a later section.

Given a non-negative function $f(P)$ not identically zero in $E^{n}$, we shall denote by $f^{*}(t), 0<t<\infty$, any non-increasing function equimeasurable with $f(P)$. If $f$ belongs to $L^{p}, 1 \leq p<\infty$, in $E^{n}$, then $f^{*}(t)$ belongs to $L^{p}$ in $0<t<\infty$ and thus is integrable over every finite interval. In this case we introduce also the function

$$
y=\beta_{f}(x)=\frac{1}{x} \int_{0}^{x} f^{*}(t) d t ; \quad x>0,
$$

which is continuous and either strictly decreasing or possibly constant in an interval $\left(0, x_{0}\right)$ and strictly decreasing for $x \geq x_{0}$. In both cases we have $\beta_{f}(x) \rightarrow 0$ as $x \rightarrow \infty$. The function inverse to $y=\beta_{f}(x)$ will be denoted by $x=\beta^{f}(y)$. If $\beta_{f}(x)$ tends to infinity as $x$ tends to zero, $\beta^{f}(y)$ is well defined for $y>0$. If $\beta_{f}(x)$ is bounded, $\beta^{f}(y)$ is well defined for all $y$ less than the least upper bound $y_{0}$ of $\beta_{f}(x)$. In this case we extend the domain of $\beta^{f}(y)$ by defining $\beta^{f}(y)=0$ for $y>y_{0}$ and $\beta^{f}\left(y_{0}\right)=\varlimsup_{y \rightarrow y_{0}} \beta^{f}(y)$.

Thus we have

$$
\begin{gathered}
\beta^{f}\left[\beta_{f}(x)\right] \geq x, \quad \beta_{f}\left[\beta^{f}(y)\right] \leq y \\
\lim _{y \rightarrow 0} \beta^{f}(y)=\infty, \quad \lim _{y \rightarrow \infty} \beta^{f}(y)=0 .
\end{gathered}
$$

We now have the following:

Lemma 1. ${ }^{1}$ Given an $f(P) \geq 0$ of $L^{p}, p \geq 1$ and any number $y>0$, there is a sequence of non-overlapping cubes $I_{k}$ such that

1 In the one-dimensional case this lemma is contained in a lemma by F. RIEsz (See [7], page 242). 


$$
y \leq \frac{1}{\left|I_{k}\right|} \int_{I_{k}} f(P) d P \leq 2^{n} y ; \quad(k=1,2, \ldots)
$$

and $f(P) \leq y$ almost everywhere outside $D_{y}=\bigcup_{k} I_{k} . \quad$ Moreover $\left|D_{y}\right| \leq \beta^{f}(y)$ and

$$
y \leq \frac{1}{\left|D_{y}\right|} \int_{D_{y}} f(P) d P \leq 2^{n} y
$$

Proof. On account of the properties of $\beta_{f}(x)$, for the given $y$ we can find an $x$ such that $\beta_{f}(x)<y$. Then over any cube $I$ of measure $x$ we have

$$
|I| \int_{I} f(P) d P \leq \int_{x}^{1} \int_{0}^{x} f^{*}(t) d \dot{t} \leq \beta_{f}(x)<y .
$$

Divide now $E^{n}$ into a mesh of cubes of measure $x$ and carry out the following process: divide each cube into $2^{n}$ equal cubes and select those where the average of the function $f(P)$ is larger than or equal to $y$. Then divide the remaining ones again in $2^{n}$ equal cubes and select those where the average of the function is larger than or equal to $y$. Continuing this process we obtain a sequence of cubes $I_{k}$ which we shall show have the required properties. First of all, we obviously have

$$
\frac{1}{\left|I_{k}\right|} \int_{I_{k}} f(P) d P \geq y
$$

Moreover, since every selected cube $I_{k}$ was obtained from dividing a cube $I$ where the average of the function $f(P)$ was less than $y$, we also have

$$
\int_{I_{k}} f(P) d P \leq \int_{I} f(P) d P \leq|I| y=\left|I_{k}\right| 2^{n} y
$$

and therefore

$$
\frac{1}{\left|I_{k}\right|} \int_{I_{k}} f(P) d P \leqq 2^{n} y
$$

Now, every point outside $D_{y}=U I_{k}$ is contained in arbitrarily small cubes over which the average of $f(P)$ is less than $y$. Therefore the derivative of the indefinite integral of $f$ cannot exist and be larger than $y$, and since $f(P)$ is almost everywhere equal to the derivative of its indefinite integral, we conclude that $f(P) \leq y$ almost everywhere outside $D_{y}$.

Finally we have 
and hence

$$
y\left|I_{k}\right| \leq \int_{I_{k}} f(P) d P \leq 2^{n} y\left|I_{i}\right|
$$

$$
y \sum_{1}^{m}\left|I_{k}\right| \leq \int_{\substack{m \\ \cup \\ 1}} f(P) d P \leq 2^{n} y \sum_{1}^{m}\left|I_{k}\right|
$$

or

$$
y \leq \frac{1}{\sum_{1}^{m}\left|I_{k}\right|} \int_{\substack{m \\ \cup}} f(P) d P \leq 2^{n} y .
$$

Since $\left|\mathbf{I}_{1}^{m} I_{k}\right|=\sum_{i}^{m}\left|I_{k}\right|$, it follows that

Therefore

$$
\sum_{1}^{m}\left|I_{k}\right| \underset{\substack{\mathfrak{u}_{1}^{m} I_{k} \\ \text { m }}}{1} f(P) d P \leq \beta_{f}\left(\sum_{I}^{m}\left|I_{k}\right|\right) .
$$

and

$$
y \leq \beta_{f}\left(\sum_{1}^{m}\left|I_{k}\right|\right)
$$

$$
\sum_{1}^{m}\left|I_{k}\right| \leq \beta^{f}(y)
$$

and letting $m$ tend to infinity we get $\left|D_{y}\right| \leq \beta^{f}(y)$. Therefore $\left|D_{y}\right|$ is finite, and repeating the argument above, replacing now $\sum_{i}^{m}\left|I_{k}\right|$ by $\sum_{1}^{\infty}\left|I_{k}\right|=\left|D_{y}\right|$, we shall finally get

$$
y \leq \mid \frac{1}{D_{y} \mid} \int_{D_{y}} f(P) d P \leq 2^{n} y .
$$

This completes the proof.

Lemma 2. Let $f \geq 0$ belong to $L^{p}, 1 \leq p \leq 2$, in $E^{n}$, and let $E_{y}$ be the set of points where the function

$$
\tilde{f}_{\lambda}(P)=\int_{E^{n}} K_{\lambda}(P-Q) f(Q) d Q
$$

exceeds $y$ in absolute value. Then

$$
\left|E_{y}\right| \leq \frac{c_{1}}{y^{2}} \int_{E^{n}}[f(P)]_{y}^{2} d P+c_{2} \beta^{f}(y),
$$

where $[f(P)]_{y}$ denotes the function equal to $f(P)$ if $f(P) \leq y$ and equal to $y$ otherwise, and $c_{1}$ and $c_{2}$ are constants independent of $\lambda$.

Proof. In order to simplify notation, every constant depending onlv on the dimension $n$ and the function $\Omega$ will be denoted by $c$ simply. 
Let $D_{y}$ be the set of Lemma 1 and define

$$
h(P)=\left\{\begin{array}{l}
\frac{1}{\left|I_{k}\right|} \int_{I_{k}} f(Q) d Q, \quad \text { if } P \in I_{k} ; \\
f(P) \text { otherwise. }
\end{array}\right.
$$

Then $f(P)=h(P)+g(P)$, with $g(P)=0$ outside $D_{y}$, and

Define now

$$
\int_{I_{k}} g(P) d P=0, \quad k=1,2, \ldots
$$

$$
\begin{aligned}
& \tilde{h}_{\lambda}(P)=\int_{E^{n}} K_{\lambda}(P-Q) h(Q) d Q \\
& \tilde{g}_{\lambda}(P)=\int_{E^{n}} K_{\lambda}(P-Q) g(Q) d Q
\end{aligned}
$$

and denote by $E_{1}$ the set of points where $\left|\tilde{h}_{\lambda}(P)\right| \geq y / 2$, and by $E_{2}$ that where $\left|\tilde{g}_{\lambda}(P)\right| \geq y / 2$.

As we have already shown (see (2a)),

$$
\int_{E^{n}}\left|\tilde{h}_{\lambda}(P)\right|^{2} d P \leq c \int_{E^{n}} h(Q)^{2} d Q
$$

where $c$ is a constant independent of $\lambda$. From this it easily follows that

$$
\left|E_{1}\right| \leq \frac{4 c}{y^{2}} \int_{E^{n}} h(Q)^{2} d Q
$$

Now, on account of the definition of $h$, we have $h(P)=f(P) \leq y$ outside $D_{y}$ and therefore $h(P)=[f(P)]_{y}$ outside $D_{y} ;$ moreover $h(P) \leq 2^{n} y$ in $D_{y}$. Therefore, denoting by $D_{y}^{\prime}$ the complement of $D_{y}$, we have

and

$$
\int_{E^{n}} h(Q)^{2} d Q=\int_{D_{y}} h(Q)^{2} d Q+\int_{D^{\prime} y} h(Q)^{2} d Q \leq 2^{2 n} y^{2}\left|D_{y}\right|+\int_{E^{n}}[f(P)]_{y}^{2} d P
$$

$$
\left|E_{1}\right| \leq \frac{c}{y^{2}} \int_{E^{n}}[f(P)]_{y}^{2} d P+c\left|D_{y}\right|
$$

To estimate the measure of $E_{2}$ we proceed as follows. Denote by $S_{k}$ the sphere with the same center as $I_{k}$, and radius equal to the diameter of $I_{k}$, and call $\bar{D}_{y}=\bigcup_{1}^{\infty} S_{k}$ and $\bar{D}_{y}^{\prime}$ its complement. Then $\left|\bar{D}_{y}\right| \leq c\left|D_{y}\right|$ and

$$
\left|E_{2}\right| \leq\left|\bar{D}_{y}\right|+\left|E_{2} \cap \bar{D}_{y}^{\prime}\right| \leq c\left|D_{y}\right|+\left|E_{2} \cap \bar{D}_{y}^{\prime}\right|
$$


Since $g(P)=0$ outside $D_{y}$, we have

$$
\tilde{g}_{\lambda}(P)=\sum_{\widehat{k}} \int_{I_{k}} g(Q) K_{\lambda}(P-Q) d Q .
$$

Let us now estimate the integral of $\left|g_{\lambda}(P)\right|$ over $\bar{D}_{y}^{\prime}$. Suppose that $P$ belongs to $\bar{D}_{y}^{\prime}$ and consider one of the cubes $I_{k}$. If $I_{k}$ has no points in common with the sphere with center at $P$ and radius $1 / \lambda$ we have

$$
\int_{i_{k}} g(Q) K_{\lambda}(P-Q) d Q=\int_{I_{k}} g(Q) K(P-Q) d Q
$$

since $K_{\lambda}=K$ outside that sphere. Since the integral of $g$ over $I_{k}$ is zero we have, furthermore,

$$
\int_{I_{k}} g(Q) K_{\lambda}(P-Q) d Q=\int_{I_{k}} g(Q)\left[K(P-Q)-K\left(P-Q_{k}\right)\right] d Q,
$$

where $Q_{k}$ is the center of $I_{k}$. Now, if $P$ is outside $S_{k}$ and $Q$ is in $I_{k}$, from the continuity properties of $\Omega$ and, by an elementary geometrical argument, we deduce that

$$
\left|K(P-Q)-K\left(P-Q_{k}\right)\right| \leq c\left|P-Q_{k}\right|^{-n} \omega\left[c\left|I_{k}\right|^{1 / n}\left|P-Q_{k}\right|^{-1}\right]{ }^{1}
$$

and therefore

$$
\left|\int_{I_{k}} g(Q) K_{\lambda}(P-Q) d Q\right| \leq c\left|P-Q_{k}\right|^{-n} \omega\left[c\left|I_{k}\right|^{1 / n}\left|P-Q_{k}\right|^{-1}\right] \int_{I_{k}}|g(Q)| d Q .
$$

On the other hand, if $I_{k}$ intersects the sphere of radius $1 / \lambda$ and center at $P$, and $P$ is outside $S_{k}, I_{k}$ is entirely contained in the sphere of radius $3 / \lambda$, and center at

1 Since this argument is going to be used repeatedly, we shall give it here. Let us denote by $R$ and $S$ the projections of $Q$ and $Q_{k}$ on the unit sphere with center at $P$. Then $K(P-Q)-$ $K\left(P-Q_{k}\right)$ can be written

$$
\frac{\Omega(R)}{|P-Q|^{n}}-\frac{\Omega(S)}{\left|P-Q_{k}\right|^{n}}=\frac{\Omega(R)-\Omega(S)}{\left|P-Q_{k}\right|^{n}}+\left\{\frac{1}{|P-Q|^{n}}-\frac{1}{\left|P-Q_{k}\right|^{n}}\right\} \Omega(R) .
$$

The second term on the right is numerically

$$
\leq \frac{c}{\left|P-Q_{k}\right|^{n}} \frac{\left|I_{k}\right|^{1 / n}}{\left|P-Q_{k}\right|} \leq \frac{c}{\left|P-Q_{k}\right|^{n}} \omega\left(\frac{\left|I_{k}\right|^{1 / n}}{\left|P-Q_{k}\right|}\right) .
$$

For the first term on the right, we have

and thus

$$
|R-S| \leq c\left|I_{k}\right|^{1 / n}\left|P-Q_{k}\right|^{-1},
$$

$$
|\Omega(R)-\Omega(S)| \leq \omega(|R-S|) \leq \omega\left(c\left|I_{k}\right|^{1 / n}\left|P-Q_{k}\right|^{-1}\right) .
$$

Collecting the results we obtain the desired inequality. 
$P$, so that, if $\gamma(t)$ is the characteristic function of the interval $(0,3)$ and $c$ is a bound for $\Omega$, we have or, for all $Q$ in $I_{k}$,

$$
\left|K_{\lambda}(P-Q)\right| \leq c \lambda^{n}
$$

$$
\left|K_{\lambda}(P-Q)\right| \leq c \lambda^{n} \gamma[\lambda|P-Q|] \text {. }
$$

From this it follows that

$$
\left|\int_{I_{k}} g(Q) K_{\lambda}(P-Q) d Q\right| \leq c \lambda^{n} \int_{I_{k}} \gamma(\lambda|P-Q|)|g(Q)| d Q
$$

and this combined with the estimate above gives

or

$$
\begin{aligned}
\left|\tilde{g}_{\lambda}(P)\right| \leq \sum_{k}\left\{c | P - Q _ { k } | ^ { - n } \omega \left[c\left|I_{k}\right|^{1 / n} \mid P\right.\right. & \left.-\left.Q_{k}\right|^{-1}\right] \int_{I_{k}}|g(Q)| d Q+ \\
& \left.+c \lambda^{n} \int_{I_{k}} \gamma(\lambda|P-Q|)|g(Q)| d Q\right\}
\end{aligned}
$$

$$
\begin{aligned}
\left|\tilde{g}_{\lambda}(P)\right| \leq & c \lambda^{n} \int_{D_{y}} \gamma(\lambda|P-Q|)|g(Q)| d Q+ \\
& +\sum_{k}\left\{c\left|P-Q_{k}\right|^{-n} \omega\left[c\left|I_{k}\right|^{1 / n}\left|P-Q_{k}\right|^{-1}\right] \int_{I_{k}}|g(Q)| d Q\right\} .
\end{aligned}
$$

Integrating this over the complement $\bar{D}_{y}^{\prime}$ of $\bar{D}_{y}$ we get (denoting by $S_{k}^{\prime}$ the complement of $S_{k}$ )

$$
\begin{aligned}
& \int_{D^{\prime} y}\left|\tilde{g}_{\lambda}(P)\right| d P \leq c \int_{D_{y}}|g(Q)| d Q \int_{E^{n}} \lambda^{n} \gamma(\lambda|P-Q|) d P+ \\
& \quad+\sum_{k}\left\{c \int_{S^{\prime} \gamma_{k}}\left|P-Q_{k}\right|^{-n} \omega\left[c\left|I_{k}\right|^{1 / n}\left|P-Q_{k}\right|^{-1}\right] d P \int_{I_{k}}|g(Q)| d Q\right\} .
\end{aligned}
$$

Now, on account of the properties of $\omega(t)$, the integrals with respect to $P$ inside the summation sign are easily seen to be less than a constant, and the inner integral in the first integral on the right is a constant, regardless of the values of $\lambda$ and $P$. Thus the last inequality reduces to

$$
\int_{\bar{D}^{\prime} y}\left|\tilde{g}_{\lambda}(P)\right| d P \leq c \int_{D_{y}}|g(Q)| d Q
$$

Now, according to the definitions of $g$ and $h$, we have

and

$$
|g(P)| \leq f(P)+h(P)
$$

$$
\int_{D_{y}}|g(P)| d P \leq \int_{D_{y}}[f(P)+h(P)] d P=2 \int_{D_{y}} f(P) d P,
$$

and by Lemma 1 the last integral does not exceed $2^{n} y\left|D_{y}\right|$. Therefore 
and

$$
\int_{D^{\prime} y}\left|\tilde{g}_{\lambda}(P)\right| d P \leq c y\left|D_{y}\right|
$$

$$
\left|E_{2} \cap \bar{D}_{y}^{\prime}\right| \leq c\left|D_{y}\right|
$$

Collecting all estimates we get

$$
\left|E_{1}\right|+\left|E_{2}\right| \leq \frac{c}{y^{2}} \int_{E^{n}}[f(P)]_{y}^{2} d P+c\left|D_{y}\right|
$$

Since $E_{y} \subset E_{1} \cup E_{2}$ and $\left|D_{y}\right| \leq \beta^{f}(y)$, Lemma 2 follows from the preceeding inequality.

Theorem 1. Let $f(P)$ belong to $L^{p}, 1<p<\infty$, in $E^{n}$, then the function

$$
\tilde{f}_{\lambda}(P)=\int_{E^{n}} K_{\lambda}(P-Q) f(Q) d Q .
$$

also belongs to $L^{p}$, and

$$
\left[\int_{E^{n}}\left|\tilde{f}_{\lambda}(P)\right|^{p} d P\right]^{1 / p} \leq A_{p}\left[\int_{E^{n}}|f(P)|^{p} d P\right]^{1 / p}
$$

where $A_{p}$ is a constant independent of $\lambda$ and $t$.

Proof. Without loss of generality we may assume that $f(P) \geq 0$. We shall start with the case $1<p<2$. According to Lemma 2,

$$
\left|E_{y}\right| \leq \frac{c_{1}}{y^{2}} \int_{E^{n}}[f(P)]_{y}^{2} d P+c_{2} \beta^{f}(y)
$$

where $E_{y}$ is the set of points where $\left|\tilde{f}_{\lambda}(P)\right|$ exceeds $y$, and $c_{1}$ and $c_{2}$ are absolute constants.

We have

$$
\int_{E^{n}}\left|\tilde{f}_{\lambda}(P)\right|^{p} d P=p \int_{0}^{\infty}\left|E_{y}\right| y^{p-1} d y
$$

and replacing on the right $\left|E_{y}\right|$ by its estimate we get

$$
\int_{E^{n}}\left|\tilde{f}_{\lambda}(P)\right|^{p} d P \leq c_{1} \int_{0}^{\infty} \frac{p y^{p-1}}{y^{2}} \int_{E^{n}}[f(P)]_{y}^{2} d P d y+c_{2} \int_{0}^{\infty} \beta^{f}(y) p y^{p-1} d y .
$$

For the first integral on the right we have

$$
c_{1} \int_{0}^{\infty} \frac{p y^{p-1}}{y^{2}} \int_{E^{n}}[f(P)]_{y}^{2} d P d y=c_{1} \int_{E^{n}} d P \int_{0}^{\infty} p y^{p-1} \frac{[f(P)]_{y}^{2}}{y^{2}} d y,
$$

7 -523804. Acta mathematica. 88. Imprimé le 29 octobre 1952. 
and since $[f(P)]_{y}=y$ for $y \leq f(P)$, and $[f(P)]_{y}=f(P)$ for $y \geq f(P)$, the right hand side of the last expression can be replaced by

$$
\begin{aligned}
& c_{1} \int_{E_{n}} d P\left[\int_{0}^{f(P)} p y^{p-1} d y+\int_{f(P)}^{\infty} p y^{p-3} f^{2}(P) d y\right]= \\
& \quad=c_{1} \int_{E^{n}}\left[f(P)^{p}+\frac{p}{2-p} f(P)^{p}\right] d P=c_{1} \frac{2}{2-p} \int_{E^{n}} f(P)^{p} d P .
\end{aligned}
$$

To estimate the second integral, we set $y=\beta_{f}(x)$ and get

Now

$$
\int_{0}^{\infty} \beta^{f}(y) p y^{p-1} d y=-\int_{0}^{\infty} x d \beta_{f}^{p}(x) \text {. }
$$

$$
x \beta_{f}^{p}(x)=\frac{1}{x^{p-1}}\left[\int_{0}^{x} f^{*}(t) d t\right]^{p} \leq \int_{0}^{x} f^{*}(t)^{p} d t,
$$

and since $f(p)$ belongs to $L^{p}$ so does $f^{*}(t)$, and $x \beta_{f}^{p}(x) \rightarrow 0$ as $x \rightarrow 0$. Therefore we have

$$
-\int_{0}^{\infty} x d \beta_{f}^{p}(x) \leq \int_{0}^{\infty} \beta_{f}^{p}(x) d x
$$

and by a familiar theorem of Hardy (See [7], p. 72) the last expression does not exceed

$$
\left(\frac{p}{p-1}\right)^{p} \int_{0}^{\infty} f^{*}(t)^{p} d t=\left(\frac{p}{p-1}\right)^{p} \int_{E^{n}} f(P)^{p} d P .
$$

Collecting all inequalities, we finally get

$$
\int_{E^{n}}\left|\tilde{f}_{\lambda}(P)\right|^{p} d P \leq\left[\frac{2 c_{1}}{2-p}+c_{2}\left(\frac{p}{p-1}\right)^{p}\right] \int_{E^{n}} f(P)^{p} d P .
$$

In the case when $f$ belongs to $L^{p}$ with $p>2$ let $g$ be any function belonging to $L^{\alpha}(1 / p+1 / q=1)$ and vanishing outside a bounded set. Then

$$
\int_{E^{n}} g(P) \tilde{f}_{\lambda}(P) d P=\int_{E^{n}} g(P) d P \int_{E^{n}} K_{\lambda}(P-Q) f(Q) d Q,
$$

and inverting the order of integration, which is justified since the double integral is absolutely convergent,

$$
\int_{E^{n}} g(P) \tilde{f}_{\lambda}(P) d P=\int_{E^{n}} f(Q) d Q \int_{E^{n}} K_{\lambda}(P-Q) g(P) d P=\int_{E^{n}} f(-Q) \tilde{g}_{\lambda}^{\prime}(Q) d Q,
$$

where $g^{\prime}(P)=g(-P)$. 
Therefore

$$
\left|\int_{E^{n}} g(P) \tilde{f}_{\lambda}(P) d P\right|=\left|\int_{E^{n}} f(-Q) \tilde{g}_{\lambda}^{\prime}(Q) d Q\right| \leq\left[\int_{E^{n}}|f|^{p} d P\right]^{1 / p}\left[\int_{E^{n}}\left|\tilde{g}_{\lambda}^{\prime}\right|^{q} d Q\right]^{1 / Q},
$$

and since $q<2$ we may replace the last integral by the corresponding integral of $|g(Q)|$ times $A_{q}^{q}$, and we get

$$
\left|\int_{E^{n}} g(P) \tilde{f}_{\lambda}(P) d P\right| \leq A_{q}\left[\int_{E^{n}}|f(Q)|^{p} d Q\right]^{1 / p}\left[\int_{E^{n}}|g(Q)|^{q} d Q\right]^{1 / q},
$$

which implies that

$$
\left[\int_{E^{n}}\left|\tilde{f}_{\lambda}(P)\right|^{p} d P\right]^{1 / p} \leq A_{q}\left[\int_{E^{n}}|f(P)|^{p} d P\right]^{1 / p}
$$

This completes the proof.

Remark. The inequality (5) leads to a very crude estimate for the least value $A_{p}^{*}$ of $A_{p}$, namely

$$
A_{p}^{*}=O\left(\frac{1}{p-1}\right)+O\left(\frac{1}{2-p}\right) ; \quad 1<p<2 .
$$

This can easily be improved to

$$
A_{p}^{*}=O\left(\frac{1}{p-1}\right) ; \quad 1<p \leq 2 .
$$

For, anyway, $A_{p}^{*}$ is finite, and so, using instead of (4) the inequality

$$
\int_{E^{n}}\left|\tilde{h}_{\lambda}\right|^{4} d P \leq A_{4} \int_{E^{n}} h^{4} d P
$$

and repeating the proof of Lemma 2, we obtain instead of (3) the inequality

$$
\left|E_{y}\right| \leq \frac{c_{1}}{y^{4}} \int_{E^{n}}[f(P)]_{y}^{4} d P+c_{2} \beta^{f}(y)
$$

for all $f \in L^{p}, \quad 1 \leq p \leq 4$ which, by an argument similar to the one used in the preceding theorem, leads to (7). Since $A_{p}=A_{q}$ for $q=\frac{p}{p-1}$, we have

$$
A_{q}=O(q) ; \quad q \geq 2 .
$$

Another way of obtaining (7) would be to apply the theorem of M. Riesz on the interpolation of linear operations (See [7], p. 198) to the two exponents $p<2<q$. 
Theorem 2. Let $f(P)$ be a function such that

$$
\int_{E^{n}}|f(P)|\left(1+\log ^{+}|f(P)|\right) d P<\infty .
$$

Then $\tilde{f}_{\lambda}$ is integrable over any set $S$ of finite measure and

$$
\int_{S}\left|\tilde{f}_{\lambda}\right| d P \leq c \int_{E^{n}}|f| d P+c \int_{E^{n}}|f| \log ^{+}\left(|S|^{\frac{n+1}{n}}|f|\right) d P+c|S|^{-\frac{1}{n}}
$$

where $c$ is a constant independent of $S$ and $\lambda$.

Proof. We may assume, without loss of generality, that $f(P) \geq 0$. Let $E_{y}$ be the set of points where $\left|\tilde{f}_{\lambda}(P)\right|>y$ and $E_{y}^{\prime}=E_{y} \cap S$. Then

$$
\int_{S}\left|\tilde{f}_{\lambda}\right| d P=\int_{0}^{\infty}\left|E_{y}^{\prime}\right| d y
$$

Now $\left|E_{y}^{\prime}\right| \leq\left|E_{y}\right|$ and $\left|E_{y}^{\prime}\right| \leq|S|$, and therefore we may write

$$
\int_{S}\left|\tilde{f}_{\lambda}\right| d P \leq \int_{0}^{y_{0}}|S| d y+\int_{y_{0}}^{\infty}\left|E_{y}\right| d y=|S| y_{0}+\int_{y_{0}}^{\infty}\left|E_{y}\right| d y
$$

$y_{0}$ being any positive number.

According to Lemma 3, we have

$$
\left|E_{y}\right| \leq \frac{c_{1}}{y^{2}} \int_{E^{n}}[f(P)]_{y}^{2} d P+c_{2} \beta^{f}(y)
$$

and from this it follows that

$$
\int_{y_{0}}^{\infty}\left|E_{y}\right| d y \leq c_{1} \int_{0}^{\infty} \frac{1}{y^{2}} d y \int_{E^{n}}[f(P)]_{y}^{2} d P+c_{2} \int_{y_{0}}^{\infty} \beta^{f}(y) d y .
$$

Now, in the proof of Theorem 1 we have shown that the first integral on the right does not exceed a constant multiple of the integral

$$
\int_{E^{n}} f(P) d P .
$$

On the other hand, if we select $y_{0}=\beta_{f}(|S|)$, the integral on the right reduces, after introducing the variable $x=\beta^{f}(y)$ and integrating by parts, to 


$$
\int_{y_{0}}^{\infty} \beta^{f}(y) d y=\int_{|S|}^{0} x d \beta_{f}(x) \leq \int_{0}^{|S|} \beta_{f}(x) d x=\int_{0}^{|S|} d x \int_{x}^{x} f^{*}(t) d t=\int_{0}^{|S|} f^{*}(t) \log |S| d t .
$$

Now, the convex functions $\Phi(x)=x \log ^{+}\left(|S|^{n+1} x\right)$ and

$$
\Psi(y)=\left\{\begin{array}{l}
y|S|^{-\frac{n+1}{n}} \text { for } 0 \leq y \leq 1, \\
e^{y-1}|S|^{-\frac{n+1}{n}} \text { for } 1 \leq y,
\end{array}\right.
$$

are conjugate in the sense of Young ${ }^{1}$, so that Young's inequality gives

$$
\begin{aligned}
\int_{0}^{|S|} f^{*}(t) \log \frac{|S|}{t} d t & =2 \int_{0}^{|S|} f^{*}(t) \frac{1}{2} \log \frac{|S|}{t} d t \leq 2 \int_{E^{n}} f \log ^{+}\left(|S|^{n+1} f\right) d P+ \\
& +2|S|^{-\frac{n+1}{n}} \int_{0}^{|S|}\left(\frac{|S|}{t}\right)^{1 / 2} d t=2 \int_{E^{n}} f \log ^{+}\left(|S|^{\frac{n+1}{n}} f\right) d P+4|S|^{-1 / n}
\end{aligned}
$$

Finally, collecting results and observing that

$$
|S| y_{0}=|S| \beta_{f}(|S|)=\int_{0}^{|S|} f^{*}(t) d t \leq \int_{E^{n}} f d P,
$$

we establish our assertion.

Theorem 3. Let $f$ be integrable in $E^{n}$. Then if $S$ is a set of finite measure we have

$$
\int_{S}\left|\tilde{f}_{\lambda}(P)\right|^{1-\varepsilon} d P \leq \frac{c}{\varepsilon}|S|^{\varepsilon}\left[\int_{E^{n}}|f(P)| d P\right]^{1-\varepsilon}
$$

where $c$ is a constant independent of $\varepsilon, S, \lambda$ and $f$.

Proof. Again we shall only consider the case when $f \geq 0$. We have

From this it follows that

$$
\left|E_{y}\right| \leq \frac{c_{1}}{y^{2}} \int_{E^{n}}[f(P)]_{y}^{2} d P+c_{2} \beta^{f}(y)
$$

$$
y\left|E_{y}\right| \leq c_{1} \int_{E^{n}}[f(P)]_{y} \frac{[f(P)]_{y}}{y} d P+c_{2} y \beta^{f}(y)
$$

\footnotetext{
1 See [7], p. 64 .
} 
Since $\frac{1}{y}[f(P)]_{y} \leq 1$, and since for $\beta^{f}(y)=x$ we have

we get

$$
y \beta^{f}(y)=x \beta_{f}(x)=\int_{0}^{x} f^{*}(t) d t \leq \int_{E^{n}} f(P) d P,
$$

$$
y\left|E_{y}\right| \leq c \int_{E^{n}} f(P) d P .
$$

If we write $E_{y}^{\prime}=E_{y} \cap S$, we have

and

$$
\left|E_{y}^{\prime}\right| \leq|S|, \quad\left|E_{y}^{\prime}\right| \leq\left|E_{y}\right|
$$

$$
\begin{aligned}
& \int_{S}\left|\tilde{f}_{\lambda}(P)\right|^{1-\varepsilon} d P=-\int_{0}^{\infty} y^{1-\varepsilon} d\left|E_{y}^{\prime}\right| \leq(1-\varepsilon) \int_{0}^{\infty} \frac{\left|E_{y}^{\prime}\right|}{y^{\varepsilon}} d y \leq \\
& \quad \leq(1-\varepsilon) \int_{0}^{y_{0}} \frac{|S|}{y^{\varepsilon}} d y+(1-\varepsilon) c\left[\int_{E^{n}} f(P) d P\right] \int_{y_{0}}^{\infty} \frac{d y}{y^{1+\varepsilon}}
\end{aligned}
$$

If we set here

$$
y_{0}=|S|^{-1} c \int_{E^{n}} f(P) d P,
$$

our assertion follows. This completes the proof.

Theorem 4. Let $\mu(P)$ be a mass-distribution that is a completely additive function of Borel set in $E^{n}$, and suppose that the total variation $V$ of $\mu$ in $E^{n}$ is finite. Then if

$$
\tilde{f}_{\lambda}(P)=\int_{E^{n}} K_{\lambda}(P-Q) d \mu(Q),
$$

over every set $S$ of finite measure we have

$$
\int_{S}\left|\tilde{f}_{\lambda}(P)\right|^{1-\varepsilon} d P \leq \frac{c}{\varepsilon}|S|^{\varepsilon} V^{1-\varepsilon} .
$$

Proof. This theorem is a straightforward consequence of the preceding one.

Let $H(P)$ be a non negative continuous function vanishing outside a bounded set and such that

$$
\int_{E^{n}} H(P) d P=1
$$

Then it is known that (see e.g. Lemma 1 in Chapter II)

$$
\tilde{f}_{\lambda}(P)=\lim _{k \rightarrow \infty} k^{n} \int_{E^{n}} H[k(P-Q)] \tilde{f}_{\lambda}(Q) d Q
$$


almost everywhere. But

$$
\begin{aligned}
k^{n} \int_{E^{n}} H[k(P-Q)] \tilde{f}_{\lambda}(Q) d Q=k^{n} \int_{E^{n}} H[k(P-Q)] d Q \int_{E^{n}} K_{\lambda}(Q-R) d \mu(R)= \\
=\int_{E^{n}} K_{\lambda}(P-Q)\left[k^{n} \int_{E^{n}} H[k(Q-R)] d \mu(R)\right] d Q
\end{aligned}
$$

and thus from the preceding theorem it follows that

$$
\int_{S}\left|k^{n} \int_{E^{n}} H[k(P-Q)] \tilde{f}_{\lambda}(Q) d Q\right|^{1-\varepsilon} d P \leq \frac{c}{\varepsilon}|S|^{\varepsilon}\left[\left.\iint_{E^{n}}\left|\int_{E^{n}} k^{n} H[k(Q-R)] d \mu(R)\right| d Q\right|^{1-\varepsilon} .\right.
$$

It is now readily seen that the last integral on the right does not exceed $r$. Therefore, substituting $V$ on the right and applying Fatou's Lemma to the left-hand side we get the theorem.

Theorem 5. Let $f(P)$ be a function in $E^{n}$ such that

$$
\int_{E^{n}}|f(P)|\left(1+\log ^{+}|P-O|+\log ^{+}|f(P)|\right) d P<\infty
$$

then for $\lambda \geq 1$ the function

$$
\tilde{F}_{\lambda}(P)=\tilde{f}_{\lambda}(P)-K_{1}(P-O) \int_{E^{n}} f(Q) d Q
$$

is integrable and

$$
\int_{E^{n}}\left|\tilde{F}_{\lambda}(P)\right| d P \leq c \int_{E^{n}}|f(P)|\left(1+\log ^{+}|P-O|+\log ^{+}|f(P)|\right) d P+c,
$$

where $c$ is a constant independent of $\lambda$ and $f$.

Proof. For the sake of simplicity of notation we shall denote any constant by $c$. Let

and

$$
f_{0}(P)=f(P) \text { if }|P-O| \leq 1,
$$

$$
f_{0}(P)=0
$$

otherwise, and $f_{k}(P)=f(P)$ if $2^{k-1}<|P-O| \leq 2^{k}, f_{k}(P)=0$ otherwise, $k=1,2, \ldots$ Let

$$
\tilde{f}_{k \lambda}(P)=\int_{E^{n}} K_{\lambda}(P-Q) f_{k}(Q) d Q
$$

and

$$
\tilde{F}_{k \lambda}=\tilde{f}_{k \lambda}-K_{1}(P-O) \int_{E^{n}} f_{k}(Q) d Q
$$


Now, if $k \geq 1$ and $S_{k}$ denotes the sphere $|P-O| \leq 2^{k+1}$, then $\left|S_{k}\right|=c 2^{(k+1) n}$, and Theorem 2 gives

$$
\begin{gathered}
\int_{S_{k}}\left|\tilde{f}_{k \lambda}\right| d P \leq c \int_{E^{n}}\left|f_{k}\right| d P+c \int_{E^{n}}\left|f_{k}\right| \log ^{+}\left(\left|S_{k}\right|^{\frac{n+1}{n}}\left|f_{k}\right|\right) d P+c\left|S_{k}\right|^{-\frac{1}{n}} \leq \\
\leq c \int_{E^{n}}\left|f_{k}\right|\left(1+\log ^{+}|P-O|+\log ^{+}\left|f_{k}\right|\right) d P+c 2^{-k-1},
\end{gathered}
$$

since $|P-O| \geq 2^{k-1}$ wherever $f_{k}(P) \neq 0$. As easily seen, this inequality, with suitable $c$, also holds for $k=0$. On the other hand,

$$
\int_{S_{k}}\left|K_{1}(P-O)\right| d P \leq c \log 2^{k+1},
$$

so that

$$
\begin{aligned}
\left|\int_{S_{k}} K_{1}(P-O) d P \int_{E^{n}} f_{k}(Q) d Q\right| & \leq c \log 2^{k+1} \int_{E^{n}}\left|f_{k}(Q)\right| d Q \leq \\
& \leq c \int_{E^{n}}\left(1+\log ^{+}|P-O|\right) \mid f_{k}(Q) d Q .
\end{aligned}
$$

This, together with the estimate for the integral of $\left|\tilde{f}_{k \lambda}(P)\right|$, gives

$$
\int_{S_{k}}\left|\tilde{F}_{k \lambda}\right| d P \leq c \int_{E^{n}}\left|f_{k}\right|\left(1+\log ^{+}|P-O|+\log ^{+}\left|f_{k}\right|\right) d P+c 2^{-k-1}
$$

Since for $\lambda \geq 1$ and $|P-Q| \geq 1$ we have $K_{\lambda}(P-Q)=K(P-Q)$, and since $f_{k}(P)$ vanishes outside $S_{k-1}$, for $P$ outside $S_{k}$ we have

$$
\begin{aligned}
\tilde{F}_{k \lambda}(P)=\int_{s_{k-1}}\left[K_{\lambda}(P-Q)\right. & \left.-K_{1}(P-O)\right] f_{k}(Q) d Q= \\
& =\int_{s_{k-1}}[K(P-Q)-K(P-O)] f_{k}(Q) d Q .
\end{aligned}
$$

Now, an argument already used (see footnote to Lemma 2) shows that, on account of the continuity condition satisfied by $\Omega(P)$, for every $P$ outside $S_{k}$ and $Q$ inside $S_{k-1}$ the following inequality holds:

$$
|K(P-Q)-K(P-O)| \leq c|P-O|^{-n} \omega\left(c 2^{k+1}|P-O|^{-1}\right) .
$$

Thus, if $S_{k}^{\prime}$ denotes the complement of $S_{k}$, we obtain

$$
\begin{aligned}
\int_{S^{\prime} k}\left|\tilde{F}_{k \lambda}\right| d P \leq & \int_{S_{k}} d P \int_{E^{n}} c|P-O|^{-n} \omega\left(c 2^{k+1}|P-O|^{-1}\right)\left|f_{k}(Q)\right| d Q= \\
& =c \int_{E^{n}}\left|f_{k}(Q)\right| d Q \int_{2^{k+1}}^{\infty} r^{-n} \omega\left(c 2^{k+1} r^{-1}\right) r^{n-1} d r=c \int_{E^{n}}\left|f_{k}(Q)\right| d Q,
\end{aligned}
$$

and collecting the results we have 


$$
\int_{E^{n}}\left|\tilde{F}_{k \lambda}\right| d P \leq c \int_{E^{n}}\left|f_{k}\right|\left(1+\log ^{+}|P-O|+\log { }^{+}\left|f_{k}\right|\right) d P+c 2^{-(k+1)} \text {. }
$$

Since $\tilde{F}_{\lambda}(P)=\sum_{0}^{\infty} \tilde{F}_{k \lambda}(P)$, the theorem follows by adding the above inequalities.

This result can be worded in a different manner. Since the functions

and

$$
\Phi(x)=x \log ^{+} \alpha x
$$

$$
\Psi(y)=\left\{\begin{array}{l}
y \alpha^{-1} \text { for } 0 \leq y \leq 1 \\
e^{y-1} \alpha^{-1} \text { for } y \geq 1
\end{array}\right.
$$

are conjugate in the sense of Young, setting $x=|f(P)|, \alpha=1+|P-O|^{n+1}$ and $y=\frac{1}{2} \log ^{+}|P-O|$, for $y \geq 1$, Young's inequality gives

$$
\begin{aligned}
\frac{1}{2}|f(P)| \log ^{+}|P-O| \leq|f(P)| & \log ^{+}\left[\left(1+|P-O|^{n+1}\right)|f|\right]+ \\
& +|P-O|^{1 / 2}\left(1+|P-O|^{n+1}\right)^{-1}
\end{aligned}
$$

so that if $|f| \log ^{+}\left[\left(1+|P-O|^{n \div 1}\right)|f|\right]$ is integrable the same is true for the product $|f| \log ^{+}|P-O|$, and since $|f| \leq|f| \log |P-O|$ for $|P-O| \geq e$, and $|f| \leq 1+$ $+|f| \log ^{+}|f|$ for $|P-O|<e$, it follows that

$$
\int_{E^{n}}|f|\left(1+\log ^{+}|P-O|+\log |f|\right) d P \leq c \int_{E^{n}}|f| \log { }^{+}\left[\left(1+|P-O|^{n+1}\right)|f|\right] d P+c,
$$

and we have the following:

Corollary. The function $\tilde{F}_{\lambda}(P)$ of the preceding theorem satisfies the inequality

$$
\int_{E^{n}}\left|\tilde{F}_{\lambda}(P)\right| d P \leq c \int_{E^{n}}|f(P)| \log ^{+}\left[\left(1+|P-O|^{n+1}\right)|f(P)|\right] d P+c .
$$

If the integral of $f$ extended over the whole space is zero, then in the last inequality we can replace $\tilde{F}_{\lambda}$ by $\tilde{f}_{\lambda}$. For $n=1$ this result reduces to a known theorem about Hilbert transforms of functions on the real line [5].

Theorem 6. Let $f(P)$ be a function bounded in $E^{n}$ and $|f(P)| \leq M$. Then the integral

$$
\tilde{F}_{\lambda}(P)=\int_{E^{n}}\left[K_{\lambda}(P-Q)-K_{1}(O-Q)\right] f(Q) d Q
$$

is absolutely convergent, and

$$
\int_{E^{n}} \Psi\left[c^{-1} M^{-1}\left|\tilde{F}_{\lambda}(P)\right|, P\right] d P \leq 1
$$

where $c$ is a constant independent of $f$ and $\lambda \geq 1$, and $\Psi(y, P)$ is the function defined by $\Psi(y, P)=y \alpha^{-1}$ for $0 \leq y \leq 1, \quad \Psi(y, P)=e^{y-1} \alpha^{-1}$ for $y \geq 1, \quad \alpha=1+|P-O|^{n+1}$. 
Proof. First we observe that for fixed $P$ and $\lambda$ the function

$$
K_{\lambda}(P-Q)-K_{1}(O-Q)
$$

is bounded. Moreover as $Q$ tends to infinity this function is of the order

$$
|Q-O|^{-n} \omega\left[c|P \rightarrow O||Q-O|^{-1}\right]
$$

and thus is absolutely integrable, and the integral of its absolute value is a function of $P$ bounded on every bounded set. Consider now the functions

and

$$
\Phi(x, P)=x \log ^{+}\left[\left(1+|P-O|^{n+1}\right) x\right]
$$

$$
\Psi(y, P)=\left\{\begin{array}{l}
y\left(1+|P-O|^{n+1}\right)^{-1} \text { for } 0 \leq y \leq 1 \\
e^{y-1}\left(1+|P-O|^{n+1}\right)^{-1} \text { for } y \geq 1
\end{array}\right.
$$

which, for fixed $P$, are conjugate in the sense of Young, and let $g(P)$ be a function vanishing outside a bounded set and such that

$$
\int_{E^{n}} \Phi(|g(P)|, P) d P \leq 1
$$

Then we have

$$
\int_{E^{n}} g(P) \tilde{F}_{\lambda}(P) d P=\int_{E^{n}} g(P) d P \int_{E^{n}}\left[K_{\lambda}(P-Q)-K_{3}(O-Q)\right] f(Q) d Q,
$$

and since the double integral is absolutely convergent we may invert the order of integration and write

$$
\int_{E^{n}} g(P) \tilde{F}_{\lambda}(P) d P=\int_{E^{n}} f(Q) d Q \int_{E^{n}}\left[K_{\lambda}(P-Q)-K_{\mathbf{1}}(O-Q)\right] g(P) d P
$$

But, according to the corollary of Theorem 5,

$$
\int_{E^{n}, E^{n}}\left|\int_{\lambda}\left[K_{\lambda}(P-Q)-K_{1}(O-Q)\right] g(P) d P\right| d Q \leq c
$$

and therefore, if $|f(P)| \leq M$, then

$$
\left|\int_{E^{n}} g(P) \tilde{F}_{\lambda}(P) d P\right| \leq c M
$$

The same conclusion holds if we multiply $g$ by any function of absolute value 1 ; therefore we also have the stronger inequality

$$
\int_{E^{n}} \lg (P)|| \tilde{F_{\lambda}}(P) \mid d P \leq c M
$$


Let us now define

$$
F_{k}(P)=\left\{\begin{array}{l}
\left|\tilde{F}_{\lambda}(P)\right|, \text { if }\left|\tilde{F}_{\lambda}(P)\right| \leq k \text { and }|P-O| \leq k \\
0 \text { otherwise, }
\end{array}\right.
$$

and denoting the function $\frac{d}{d x} \Psi(x, P)$ by $\Psi^{\prime}(x, P)$ let us also define

$$
g_{k}(P)=\left\{\begin{array}{l}
\Psi^{\prime}\left[c^{-1} \beta M^{-1} F_{k}(P), P\right] \text { for } F_{k}(P) \neq 0 \\
0 \text { for } F_{k}(P)=0
\end{array}\right.
$$

where $c$ is the same as in $(7 \mathrm{a})$ and where, assuming that $F_{k} \neq 0$, we select the constant $\beta$ in such a way that

$$
\int_{E^{n}} \Phi\left(g_{k}(P), P\right) d P=1
$$

Then (see [7], p. 64) Young's inequality degenerates into equality,

$$
g_{k}(P) \cdot\left[c^{-1} \beta M^{-1} F_{k}(P)\right]=\Phi\left(g_{k}, P\right)+\Psi\left(c^{-1} \beta M^{-1} F_{k}, P\right),
$$

and integrating with respect to $P$ we get

$$
\begin{gathered}
c^{-1} \beta M^{-1} \int_{E^{n}} g_{k}(P) F_{k}(P) d P=\int_{E^{n}} \Phi\left(g_{k}, P\right) d P+\int_{E^{n}} \Psi\left(c^{-1} \beta M^{-1} F_{k}, P\right) d P= \\
=1+\int_{E^{n}} \Psi\left[c^{-1} \beta M^{-1} F_{k}(P), P\right] d P .
\end{gathered}
$$

But we also have

$$
\int_{E^{n}} g_{k}(P) F_{k}(P) d P=\int_{E^{n}} g_{k}(P)\left|\tilde{F}_{\lambda}(P)\right| d P \leq c M
$$

Thus we get

$$
1+\int_{E^{n}} \Psi\left[c^{-1} \beta M^{-1} F_{k}(P), P\right] d P \leq \beta
$$

This implies, first of all, that $\beta \geq 1$ and secondly that

$$
\int_{E^{n}} \frac{1}{\beta} \Psi\left[c^{-1} \beta M^{-1} F_{k}(P), P\right] d P \leq 1
$$

Now, since $\Psi(x, P)$ is convex, increasing and vanishes for $x=0$, and since $\beta \geq 1$, we have

$$
\frac{1}{\beta} \Psi\left[c^{-1} \beta \bar{M} M^{-1} F_{k}(P), P\right] \geq \Psi\left[c^{-1} M^{-1} F_{k}(P), P\right]
$$

and from this and the inequality above it follows that 


$$
\int_{E^{n}} \Psi\left[c^{-1} M^{-1} F_{k}(P), P\right] \leq 1,
$$

a relation which also holds for $F_{k} \equiv 0$. Finally since $F_{k}(P) \rightarrow\left|\tilde{F}_{\lambda}(P)\right|$ as $k \rightarrow \infty$, an application of Fatou's lemma establishes our assertion.

Theorem 7. Let $f(P)$ belong to $L^{p}, 1<p<\infty$ then

$$
\tilde{f}_{\lambda}(P)=\int_{E^{n}} K_{\lambda}(P-Q) f(Q) d Q
$$

converges in the mean of order $p$ as $\lambda \rightarrow \infty$, to a function $\tilde{f}(P)$ of $L^{p}$ in $E^{n}$.

If $f(P)$ is such that

$$
\int_{E^{n}}|f| \log ^{+}\left[\left(1+|P-O|^{n+1}\right)|f|\right] d P<\infty,
$$

then $\tilde{F}_{\lambda}(P)$ converges in the mean of order 1 to a /unction $\tilde{F}(P)$ integrable in $E^{n}$.

Proof. If $g(P)$ is a function with continuous first derivatives and vanishing outside a bounded set, then

$$
\tilde{g}_{\lambda}(P)=\int_{E^{n}} K_{\lambda}(P-Q) g(Q) d Q
$$

converges uniformly to a function $\tilde{g}(P)$ and moreover, outside a bounded set, $\tilde{g}_{\lambda}(P)=\tilde{g}(P)$ for $\lambda \geq 1$. This is easy to verify on account of the properties of $K_{\lambda}(P-Q)$, of the differentiability of $g$, and of the fact that

$$
\tilde{g}_{\lambda}(P)=\int_{E^{n}} K_{\lambda}(P-Q)[g(Q)-g(P)] d Q .
$$

Therefore, not only $\tilde{g}_{\lambda}(P) \rightarrow \tilde{g}(P)$ but also

$$
\int_{E^{n}}\left|\tilde{g}_{\lambda}(P)-\tilde{g}(P)\right|^{p} d P \rightarrow 0
$$

as $\lambda \rightarrow \infty$, for any $p \geq 1$.

Let now $f$ be a function of $L^{p}, 1<p<\infty$. Given any $\varepsilon>0$ there exists a function $g$ with continuous first derivatives and vanishing outside a bounded set such that

$$
\left[\int_{E^{n}}|f(P)-g(P)|^{p} d P\right]^{1 / p}<\varepsilon .
$$

Then, if $h=f-g$, 
and

$$
\tilde{f}_{\lambda}=\tilde{g}_{\lambda}+\tilde{h}_{\lambda}
$$

$$
\left[\int_{E^{n}}\left|\tilde{f}_{\lambda}-\tilde{f}_{\mu}\right|^{p} d P\right]^{1 / p} \leq\left[\int_{E^{n}}\left|\tilde{g}_{\lambda}-\tilde{g}_{\mu}\right|^{p} d P\right]^{1 / p}+\left[\int_{E^{n}}\left|\tilde{h}_{\lambda}-\tilde{h}_{\mu}\right|^{p} d P\right]^{1 / p} .
$$

Now, since

$$
\left[\int_{E^{n}}|h|^{p} d P\right]^{1 / p}<\varepsilon
$$

Theorem 1 gives

$$
\left[\int_{E^{n}}\left|\tilde{h}_{\lambda}\right|^{p} d P\right]^{1 / p}<A_{p} \varepsilon
$$

and thus we get

$$
\left[\int_{E^{n}}\left|\tilde{f}_{\lambda}-\tilde{f}_{\mu}\right|^{p} d P\right]^{1 / p}<\left[\int_{E^{n}}\left|\tilde{g}_{\lambda}-\tilde{g}_{\mu}\right|^{p} d P\right]^{1 / p}+2 A_{p} \varepsilon
$$

As $\lambda$ and $\mu$ tend to infinity, the integral on the right tends to zero; therefore for $\lambda$ and $\mu$ large we shall have

$$
\left[\int_{E^{n}}\left|\tilde{f}_{\lambda}-\tilde{f}_{\mu}\right|^{p} d P\right]^{1 / p}<3 A_{p} \varepsilon
$$

and, since $\varepsilon$ is arbitrary, the first part of the theorem is established.

For the second part we shall begin by showing that, given any $\varepsilon>0$, there exists a function $g$ with continuous first derivatives and vanishing outside a bounded set, such that

$$
\int_{E^{n}}\left|\frac{f-g}{\varepsilon}\right| \log ^{+}\left[\left(1+|P-O|^{n+1}\right) \frac{|f-g|}{\varepsilon}\right] d P \leq 1 .
$$

For let $S$ be a sphere with center at $O$ and so large that

$$
\int_{S^{\prime}}\left|\frac{f}{\varepsilon}\right| \log ^{+}\left[\left(1+|P-O|^{n+1}\right)\left|\frac{f}{\varepsilon}\right|\right] d P \leq \frac{1}{2} .
$$

For the points $P$ inside $S$ we shall have

$$
x \log ^{+}\left[\left(1+|P-O|^{n+1}\right) x\right] \leq c x^{2},
$$

for all $x \geq 0$ and a suitable $c$.

We now select $k$ so large that

$$
\int_{S}\left|\frac{f-[f]_{k}}{\varepsilon / 2}\right| \log ^{+}\left[\left(1+|P-O|^{n+1}\right)\left|\frac{f-[f]_{k}}{\varepsilon / 2}\right|\right] d P \leq \frac{1}{2}
$$

and then $g$ in such a way that $g=0$ outside $S$ and 


$$
\int_{S}\left|\frac{g-[f]_{k}}{\varepsilon / 2}\right|^{2} d P \leq \frac{1}{2 c}
$$

Then

$$
\int_{S}\left|\frac{g-[f]_{k}}{\varepsilon / 2}\right| \log ^{+}\left[\left(1+|P-O|^{n+1}\right)\left|\frac{g-[f]_{k}}{\varepsilon / 2}\right|\right] d P \leq \frac{1}{2}
$$

and, applying Jensen's inequality,

$$
\int_{S}\left|\frac{f-g}{\varepsilon}\right| \log ^{+}\left[\left(1+|P-O|^{n+1}\right)\left|\frac{f-g}{\varepsilon}\right|\right] d P \leq \frac{1}{2},
$$

which in conjunction with (9) gives (8).

Let now $h(P)=f(P)-g(P)$; then

$$
\int_{E^{n}}\left|\tilde{F}_{\lambda}-\tilde{F}_{\mu}\right| d P \leq \int_{E^{n}}\left|\tilde{G}_{\lambda}-\tilde{G}_{\mu}\right| d P+\int_{E^{n}}\left|\tilde{H}_{\lambda}-\tilde{H}_{\mu}\right| d P,
$$

where

$$
\tilde{F}_{\lambda}(P)=\int_{E^{n}} K_{\lambda}(P-Q) f(Q) d Q-K_{1}(P-O) \int_{E^{n}} f(Q) d Q
$$

and similarly for $\tilde{G}_{\lambda}$ and $\tilde{H}_{\lambda}$.

Now

$$
\int_{E^{n}}\left|\tilde{G}_{\lambda}-\tilde{G}_{\mu}\right| d P \rightarrow 0
$$

as $\lambda$ and $\mu$ tend to infinity. On the other hand, since

$$
\int_{E^{n}}\left|\frac{h}{\varepsilon}\right| \log ^{+}\left[\left(1+|P-O|^{n+1}\right)\left|\frac{h}{\varepsilon}\right|\right] d P \leq 1,
$$

by the corollary of Theorem 5 we have

$$
\int_{E^{n}}\left|\frac{\tilde{H}_{\lambda}}{\varepsilon}\right| d P \leq 2 c
$$

therefore for $\lambda$ and $\mu$ large we shall have

$$
\int_{E^{n}}\left|\tilde{F}_{\lambda}-\tilde{F}_{\mu}\right| d P \leq 4 c \varepsilon
$$

and since $\varepsilon$ is arbitrary the theorem is established.

Remarks $1^{\circ}$. Under the assumptions of Theorem 2 , the function $\tilde{f}_{\lambda}(P)$ converges to a limit $\tilde{f}(P)$, in the mean of order 1 , over every set of finite measure. Under the assumption $(7 \mathrm{~b})$, this mean convergence holds over $E^{n}$, but, unless $\int_{E^{n}} f d P=0$ (or $K \equiv 0$ ), neither $\tilde{f}_{\lambda}$ nor $\tilde{f}$ are of the class $L$. 
$2^{\circ}$. Under the assumptions of Theorem 3 , the function $\tilde{f}_{\lambda}(P)$ converges to $\tilde{f}(P)$, in the mean of order $1-\varepsilon$, over any set of finite measure.

$3^{\circ}$. If $f(P)$ satisfies the assumptions of Theorem 6 and, in addition, vanishes in the neighborhood of infinity, the function exp $\left\{c^{-1} M^{-1} \tilde{f}(P)\right\}$ is integrable over any bounded set $S$. If $f$ is also continuous, $\exp k|f|$ is integrable over $S$ for any $k>0$.

\section{CHAPTER II.}

\section{The pointwise convergence of the singular integrals.}

In this section we shall investigate the convergence of the singular integrals at individual points. In the case where $f(P)$ belongs to $L^{p}, p>1$, we shall prove that the singular integrals converge almost everywhere and that moreover they are dominated by a function of $L^{p}$, uniformly in $\lambda$. On the other hand, we shall show that the pointwise limit still exists almost everywhere even if the function $f(P)$ is replaced by a completely additive function of Borel set of finite total variation.

We shall begin by proving two lemmas.

Lemma 1. Let $N(P)$ be a function in $E^{n}$ and suppose that

$$
|N(P)| \leq \varphi(|P-O|) \text {. }
$$

where $\varphi(x)$ is a decreasing function of $x$ such that

$$
\int_{E^{n}} \varphi(|P-O|) d P<\infty .
$$

Then, if $f(P)$ is a function of $L^{p}, 1 \leq p<\infty$ we have

$$
\lim _{\lambda \rightarrow \infty} \lambda^{n} \int_{E^{n}} N[\lambda(P-Q)] f(Q) d Q=f(P) \int_{E^{n}} N(Q) d Q
$$

at every point $P$ of the Lebesgue set of $f(P)$.

(One says that $P$ is a Lebesgue point for $f$ if the derivative of $\int|f(P)-f(Q)| d Q$ is equal to zero at $P$. This implies in particular that the derivative of $\int f(Q) d Q$ at $P$ is $f(P))$.

Proof. Let $P$ be a point of the Lebesgue set of $f(P)$ and let $I(\varrho)$ be the integral of $|f(P)-f(Q)|$ over the sphere with center at $P$ and radius $\varrho$. Then $I(\varrho) \varrho^{-n}$ is a bounded function and tends to zero as $\varrho$ tends to zero. 
We have

$$
\begin{array}{r}
\lambda^{n} \int_{E^{n}} N[\lambda(P-Q)] f(Q) d Q=\lambda^{n} \int_{E^{n}} N[\lambda(P-Q)][f(Q)-f(P)] d Q+ \\
+f(P) \int_{E^{n}} \lambda^{n} N[\lambda(P-Q)] d Q
\end{array}
$$

and the second integral on the right is equal to $f(P) \int_{E^{n}} N(Q) d Q$ so that if we show that the first integral tends to zero as $\lambda \rightarrow \infty$ the lemma will be established. Now, this integral is in absolute value less than or equal to $\lambda^{n} \int_{0}^{\infty} \varphi(\lambda \varrho) d I(\varrho)$, and thus it suffices to show that the latter tends to zero.

Since $\varphi(\varrho)$ is decreasing and

$$
J=\int_{0}^{\infty} \varphi(\varrho) \varrho^{n-1} d \varrho<\infty
$$

$\varphi(\varrho) \varrho^{n}$ tends to zero as $\varrho \rightarrow 0$ and $\varrho \rightarrow \infty$. On the other hand, we have $I(\varrho) \varrho^{-n} \leq c$ where $c$ is a constant, or $I(\varrho) \leq c \varrho^{n}$, so that integrating by parts we have

$$
\lambda^{n} \int_{0}^{\infty} \varphi(\lambda \varrho) d I(\varrho)=-\lambda^{n} \int_{0}^{\infty} I(\varrho) d \varphi(\lambda \varrho)
$$

and since $\varphi$ is a decreasing function, if $c(\delta)$ denotes the least upper bound of $I(\varrho) \varrho^{-n}$ in $0 \leq \varrho \leq \delta$ we can write

$$
\begin{aligned}
& -\lambda^{n} \int_{0}^{\infty} I(\varrho) d \varphi(\lambda \varrho) \leq-\lambda^{n} c(\delta) \int_{0}^{\delta} \varrho^{n} d \varphi(\lambda \varrho)-\lambda^{n} c \int_{\delta}^{\infty} \varrho^{n} d \varphi(\lambda \varrho) \leq \\
& \leq n c(\delta) \int_{0}^{\delta} \lambda^{n} \varphi(\lambda \varrho) \varrho^{n-1} d \varrho+n c \int_{\delta}^{\infty} \lambda^{n} \varphi(\lambda \varrho) \varrho^{n-1} d \varrho+\lambda^{n} c \delta^{n} \varphi(\lambda \delta)= \\
& =n c(\delta) \int_{0}^{\lambda \delta} \varrho^{n-1} \varphi(\varrho) d \varrho+n c \int_{\lambda \delta}^{\infty} \varrho^{n-1} \varphi(\varrho) d \varrho+c \lambda^{n} \delta^{n} \varphi(\lambda \delta) .
\end{aligned}
$$

Now as $\lambda \rightarrow \infty$ the two last terms tend to zero and the first remains less than $n c(\delta) J$. Therefore

$$
\varlimsup_{\lambda \rightarrow \infty} \lambda^{n} \int_{0}^{\infty} \varphi(\lambda \varrho) d I(\varrho) \leq n c(\delta) J
$$

and since $c(\delta) \rightarrow 0$ as $\delta \rightarrow 0$, we have

$$
\lim _{\lambda \rightarrow \infty} \lambda^{n} \int_{0}^{\infty} \varphi(\lambda \varrho) d I(\varrho)=0,
$$

and the lemma is established. 
Lemma 2. Under the assumptions of Lemma 1, the function

$$
\lambda^{n} \int_{E^{n}} N[\lambda(P-Q)] f(Q) d Q
$$

converges to $f(P) \int_{E^{n}} N(Q) d Q$ in the mean of order $p$.

Proof. Let us denote by $\hat{f}_{\lambda}$ the integral in question and by $\hat{f}$ its pointwise limit. If $f$ is bounded and vanishes outside a sphere of radius $r$ with center at 0 , $\hat{f}_{\lambda}$ is bounded for $|P-O|<2 r$, and less than $c \lambda^{n} \varphi\left(|P-O| \frac{\lambda}{2}\right)$ for $|P-O| \geq 2 r$, where $c$ is a constant. Inside the sphere $|P-O|<2 r, \hat{f_{\lambda}}$ converges to $\hat{f}$ boundedly and almost everywhere. Thus over the sphere $|P-O|<2 r$ we have

$$
\lim _{\lambda \rightarrow \infty} \int\left|\hat{f}_{\lambda}-\hat{f}\right|^{p} d P=0
$$

Over the exterior of the sphere we have

$$
\int\left|\hat{f}_{\lambda}\right|^{p} d P \leq c \lambda^{n p} \int \varphi\left(|P-O| \frac{\lambda}{2}\right)^{p} d P \leq c\left[\lambda^{n} \varphi(r \lambda)\right]^{p-1} \lambda^{n} \int \varphi\left(|P-O| \frac{\lambda}{2}\right) d P,
$$

and the last expression tends to zero as $\lambda \rightarrow \infty$.

To extend the result to general functions we observe first that, with $1 / p+1 / q=1$,

$$
\begin{aligned}
& \int_{E^{n}}\left|\hat{t}_{\lambda}\right|^{p} d P=\int_{E^{n}} d P\left|\int_{E^{n}} \lambda^{n} N[\lambda(P-Q)] f(Q) d Q\right|^{p} \leq \\
& \leq \int_{E^{n}} d P\left|\int_{E^{n}}\left\{\lambda^{n} \varphi(\lambda|P-Q|)\right\}^{1 / q}\left\{\lambda^{n} \varphi(\lambda|P-Q|)\right\}^{1 / p}\right| f(Q)|d Q|^{p} \leq \\
& \leq \int_{E^{n}} d P\left\{\int_{E^{n}} \lambda^{n} \varphi(\lambda|P-Q|) d Q\right\}^{p / q}\left\{\int_{E^{n}} \lambda^{n} \varphi(\lambda|P-Q|)|f|^{p} d Q\right\},
\end{aligned}
$$

and since

$$
\int_{E^{n}} \lambda^{n} \varphi(\lambda|P-Q|) d P=\int_{E^{n}} \lambda^{n} \varphi(\lambda|P-Q|) d Q=\int_{E^{n}} \varphi(|P-O|) d P,
$$

we obtain

$$
\left[\int_{E^{n}}\left|\hat{f}_{\lambda}(P)\right|^{p} d P\right]^{1 / p} \leq c\left[\int_{E^{n}}|f(Q)|^{p} d Q\right]^{1 / p}
$$

where $c=\int_{E^{n}} \varphi(|P-O|) d P$.

Thus, given $f \in L^{p}$, we may split $f$ into two functions, $f=g+h$, where $g$ is bounded and vanishes outside a bounded set and $\left[\int_{E^{n}}|h|^{p} d P\right]^{1 / p}<\varepsilon$. Then we have $\hat{f}_{\lambda}=\hat{h}_{\lambda}+\hat{g}_{\lambda}$ and

8-523804. Acta mathematica. 88. Imprimé le 29 octobre 1952. 


$$
\left[\int_{E^{n}}\left|\hat{f}_{\lambda}-\hat{f}\right|^{p} d P\right]^{1 / p} \leq\left[\int_{E^{n}}\left|\hat{h}_{\lambda}-\hat{h}\right|^{p} d P\right]^{1 / p}+\left[\int_{E^{n}}\left|\hat{g}_{\lambda}-g\right|^{p} d P\right]^{1 / p} .
$$

As $\lambda \rightarrow \infty$, the last integral tends to zero, and since

$$
\left[\int_{E^{n}}\left|\hat{h}_{\lambda}-h\right|^{p} d P\right]^{1 / p} \leq\left[\int_{E^{n}}\left|\hat{h}_{\lambda}\right|^{p} d P\right]^{1 / p}+\left[\int_{E^{n}}|h|^{p} d P\right]^{1 / p} \leq(c+1) \varepsilon,
$$

for $\lambda$ sufficiently large we shall have

$$
\left[\int_{E^{n}}\left|\hat{f}_{\lambda}-f\right|^{p} d P\right]^{1 / p} \leq(c+2) \varepsilon
$$

and the assertion follows from the fact that $\varepsilon$ is arbitrary.

Lemma 3. Let $N_{1}(P)$ be equal to 1 in the sphere of volume 1 and center at 0 , and zero elsewhere; and let $\bar{f}(P)$ be defined by

$$
\bar{f}(P)=\sup _{\lambda} \lambda^{n} \int_{E^{n}} N_{1}[\lambda(P-Q)]|f(Q)| d Q .
$$

Then, if $f$ belongs to $L^{p}, 1<p<\infty$, the same is true of $\bar{f}(P)$ and

$$
\int_{E^{n}} \bar{f}(P)^{p} d P \leq c \int_{E^{n}}|f(P)|^{p} d P,
$$

where $c$ is a constant depending on $p$ only. If $|f| \log ^{+}|f|$ is integrable then $\bar{f}(P)$ is locally integrable, and over every set $S$ of finite measure we have

$$
\int_{S} \bar{f}(P) d P \leq c \int_{S}|f(P)| d P+c \int_{S} \log ^{+}\left[|S|^{\frac{n+1}{n}}|f(P)|\right] d P+c|S|^{-\frac{1}{n}} .
$$

In general, under the assumptions of Lemma 1 we have

$$
\sup _{\lambda}\left|\lambda^{n} \int_{E^{n}} N[\lambda(P-Q)] f(Q) d Q\right| \leq \bar{f}(P) \int_{E^{n}} \varphi(|P-O|) d P,
$$

where $\bar{f}(P)$ is the function defined above.

Proof. Without loss of generality we may assume that $f(P) \geq 0$. Denote by $\bar{D}_{y}$ the set of points where $\bar{f}(P)>y$. The sets $\bar{D}_{y}$ are closely related to the sets $D_{y}$ of Lemma 1 of the preceding chapter and we shall refer part of the argument to that lemma and Theorems 1 and 2 in that section.

Let $P \in \bar{D}_{y}$. On account of the definition of $\bar{D}_{y}$ then there exists a sphere with center at $P$ over which the average of $f(Q)$ is larger than $y$. Suppose that $r$ is the radius of this sphere and consider a subdivision of the space in equal cubes of edge not less 
than $r / 2$ and less than $r$, as in Lemma 1 of the preceding chapter. Consider all the cubes in the subdivision intersecting the sphere. Their total measure or volume does not exceed a fixed positive number $1 / \alpha$ (depending only on the dimension of the space) times the volume of the sphere. Therefore the average of $f(P)$ over the union of those cubes is larger than $\alpha y$, and hence there exists at least one cube where the average of the function exceeds $\alpha y$, and which is therefore contained in $D_{a y}$. If we define $D_{y}^{*}$ to be the set obtained from $D_{y}$ by enlarging five times the edges of the cubes whose union is $D_{y}$, while keeping their centers and orientation fixed, it will turn out that the center of our sphere, that is $P$, is contained in $D_{a y}^{*}$. But since $P$ is an arbitrary point of $\bar{D}_{y}$ it follows that $\bar{D}_{y} \subset D_{a y}^{*}$ and thus

$$
\left|\bar{D}_{y}\right| \leq\left|D_{\alpha y}^{*}\right| \leq 5^{n}\left|D_{\alpha y}\right| \leq 5^{n} \beta^{f}(\alpha y) .
$$

From this the assertion on the class of $\bar{f}(P)$ would follow as in Theorems 1 and 2 of the preceding chapter. We omit the argument because it would be a mere repetition.

In the general case we have

$$
\left|\int_{E^{n}} \lambda^{n} N[\lambda(P-Q)] f(Q) d Q\right| \leq \int_{E^{n}} \lambda^{n} \varphi(\lambda|P-Q|)|f(Q)| d Q
$$

and, denoting by $I(\varrho)$ the integral of $|f(Q)|$ over the sphere with center at $P$ and radius $\varrho$, the last integral can be written as follows

$$
\int_{0}^{\infty} \lambda^{n} \varphi(\lambda \varrho) d I(\varrho)
$$

Now, if $v_{n}$ denotes the volume of the sphere of radius 1 , we have $I(\varrho) \leq v_{n} \varrho^{n} \bar{f}(P)$, and since $\varphi(\lambda \varrho) \varrho^{n} \rightarrow 0$ as $\varrho$ tends to zero or infinity, we can integrate the last integral by parts and write

$$
\begin{gathered}
\int_{0}^{\infty} \lambda^{n} \varphi(\lambda \varrho) d I(\varrho)=-\lambda^{n} \int_{0}^{\infty} I(\varrho) d \varphi(\lambda \varrho) \leq-\lambda^{n} \int_{0}^{\infty} v_{n} \varrho^{n} \bar{f}(P) d \varphi(\lambda \varrho)= \\
=\bar{f}(P) \int_{0}^{\infty} \lambda^{n} \varphi(\lambda \varrho) d v_{n} \varrho^{n}=\bar{f}(P) \int_{E^{n}} \varphi(|P-O|) d P .
\end{gathered}
$$

This completes the argument.

Remark. The first part of Lemma 3 concerning $N$, and for $n=1$ is the very well known result of Hardy and Littlewood (see [7], p. 244). In the general case spheres with center at $P$ can be replaced by cubes with center at $P$ (which also 
follows from the result concerning the function $N$ ). In this case, and for $p>1$, the case of general $n$ can easily be deduced from the original Hardy-Littlewood result by induction and the cubes can even be replaced by arbitrary $n$-dimensional intervals with fixed orientation. (See [4]). However, the case of $|f| \log ^{+}|f|$ integrable seems to require a special treatment.

An alternative proof for the latter case was communicated to us by Professor B. Jessen. He pointed out that it is enough to prove the result for differentiation with respect to a net of cubes, and that in this case the result for general $n$ is deducible from the result for $n=1$ by a measure preserving mapping of $E^{n}$ onto $E^{1}$, which transforms the cubes of the net into intervals of $E^{1}$.

Theorem 1. If $f(P)$ belongs to $L^{p}, 1<p<\infty$ then

$$
\tilde{f}_{\lambda}(P)=\int_{E^{n}} K_{\lambda}(P-Q) f(Q) d Q
$$

converges almost everywhere to a function $\tilde{f}(P)$ as $\lambda \rightarrow \infty$. Moreover the function $\sup _{\lambda}\left|\tilde{f}_{\lambda}(P)\right|$ belongs to $L^{p}$ and

$$
\int_{E^{n}} \sup _{\lambda}\left|\tilde{f}_{\lambda}(P)\right|^{p} d P \leq c \int_{E^{n}}|f(P)|^{p} d P
$$

$c$ being a constant which depends on $p$ and on the kernel $K_{\lambda}$ only.

Proof. In the preceding chapter we have shown that $\tilde{f}_{\lambda}$ converges in the mean of order $p$ to a function $\tilde{f}$ of $L^{p}$.

Let $H(P)$ be a non negative continuous function with everywhere continuous first derivatives, vanishing outside the sphere with center at 0 and radius 1 , and such that

$$
\int_{E^{n}} H(P) d P=1
$$

Then the function

$$
\hat{f}_{\mu}(P)=\mu^{n} \int_{E^{n}} H[\mu(P-Q)] \tilde{f}(Q) d Q
$$

converges almost everywhere to $\tilde{f}$ as $\mu \rightarrow \infty$ and moreover

$$
\int_{E^{n}} \sup _{\mu}\left|\hat{f}_{\mu}\right|^{p} d P \leq c \int_{E^{n}}|\tilde{f}|^{p} d P \leq c^{\prime} \int_{E^{n}}|f|^{p} d P,
$$

$c$ and $c^{\prime}$ being two constants independent of $f$. Since $\tilde{f}_{\lambda}$ converges in the mean of order $p$ to $\tilde{f}$ and $H[\mu(P-Q)]$ belongs to all classes $L^{\mathcal{D}}$ we have 


$$
\begin{aligned}
\hat{f}_{\mu}(P)=\lim _{\lambda \rightarrow \infty} \int_{E^{n}} \mu^{n} H[\mu(P-Q)] \tilde{f}_{\lambda}(Q) d Q= \\
\quad=\lim _{\lambda \rightarrow \infty} \int_{E^{n}} \mu^{n} H[\mu(P-Q)] \int_{E^{n}} K_{\lambda}(Q-S) f(S) d S,
\end{aligned}
$$

or, interchanging the order of integration,

$$
\hat{f}_{\mu}(P)=\lim _{\lambda \rightarrow \infty} \int_{E^{n}} f(S) d S\left[\int_{E^{n}} \mu^{n} H[\mu(P-Q)] K_{\lambda}(Q-S) d Q\right] .
$$

Now, since $H[\mu(P-Q)]$ belongs to all classes $L^{p}$ and has continuous first derivatives, as $\lambda \rightarrow \infty$ the inner integral converges pointwise and in the mean of order $q=\frac{p}{p-1}$, so that we can pass to the limit under the integral sign and write

$$
\hat{f}_{\mu}(P)=\int_{E^{n}} f(S)\left[\lim _{\lambda \rightarrow \infty} \int_{E^{n}} \mu^{n} H[\mu(P-Q)] K_{\lambda}(Q-S) d Q\right] d S .
$$

Since $K_{\lambda}(Q-S)=\lambda^{n} K_{1}[\lambda(Q-S)]$, introducing the variable $\mu(P-Q)=R$ the inner integral can be written as

and, setting

$$
\int_{E^{n}} \mu^{n} H(R) \frac{\lambda^{n}}{\mu^{n}} K_{\mathrm{I}}\left\{\frac{\lambda}{\mu}[(P-S) \mu-R]\right\} d R
$$

we have

$$
\tilde{H}(P)=\lim _{\lambda \rightarrow \infty} \int_{E^{n}} \lambda^{n} K_{1}[\lambda(P-Q)] H(Q) d Q
$$

$$
\lim _{\lambda \rightarrow \infty} \int_{E^{n}} \mu^{n} H[\mu(P-Q)] K_{\lambda}(Q-S) d Q=\mu^{n} \tilde{H}[\mu(P-S)]
$$

and

$$
\hat{f}_{\mu}(P)=\int_{E^{n}} \mu^{n} \tilde{H}[\mu(P-S)] f(S) d S
$$

Now $H(P)$ has continuous first derivatives and vanishes outside the sphere with center at 0 and radius 1 and therefore $\tilde{H}(P)$ is bounded and, for $|P-O| \geq 2$,

$$
\tilde{H}(P)=\int_{E^{n}} K_{1}(P-Q) H(Q) d Q
$$

On the other hand, since

$$
\int_{E^{n}} H(P) d P=1,
$$

for $|P-O| \geq 2$ we have also 


$$
\tilde{H}(P)-K_{1}(P-O)=\int_{E^{n}}\left[K_{1}(P-Q)-K_{1}(P-O)\right] H(Q) d Q
$$

and on account of the conditions satisfied by the function $\Omega$ in the definition of $K_{\lambda}(P-Q)$ it follows that, for large $|P-O|$ and $|Q-O| \leq 1$,

and thus

$$
\left|K_{1}(P-Q)-K_{1}(P-O)\right| \leq c|P-O|^{-n} \omega\left(|P-O|^{-1}\right)
$$

$$
\left|\tilde{H}(P)-K_{1}(P-O)\right| \leq c|P-O|^{-n} \omega\left(|P-O|^{-1}\right),
$$

where $c$ is a constant. Now $K_{1}(P-O)$ is bounded, as well as $\tilde{H}(P)$, so that for all $P$ the inequality

$$
\left|\tilde{H}(P)-K_{1}(P-O)\right| \leq c \min \left\{1 ;|P-O|^{-n} \omega\left(|P-O|^{-1}\right)\right\}
$$

will hold, $c$ being again a constant.

Now

$$
\hat{f}_{\mu}(P)-\tilde{f}_{\mu}(P)=\int_{E^{n}} \mu^{n}\left\{\tilde{H}\left[\mu(P-Q)-K_{1}[\mu(P-Q)]\right\} f(Q) d Q .\right.
$$

Thus from Lemmas 1 and 3 it follows that $\hat{f}_{\mu}(P)-\tilde{f}_{\mu}(P)$ converges almost everywhere and that

$$
\int_{E^{n}} \sup _{\mu}\left|\hat{f}_{\mu}(P)-\tilde{f}_{\mu}(P)\right|^{p} d P \leq c \int_{E^{n}}|f(P)|^{p} d P .
$$

Since

almost everywhere, and

$$
\lim _{\mu \rightarrow \infty} \hat{f}_{\mu}(P)=\tilde{f}(P)
$$

$$
\int_{E^{n}} \sup _{\mu}\left|\hat{f}_{\mu}(P)\right|^{p} d P \leq c^{\prime} \int_{E^{n}}|f(P)|^{p} d P
$$

and $\tilde{f}_{\mu} \rightarrow \tilde{f}$ in mean of order $p$, the theorem follows.

Theorem 2. Let $\mu(P)$ be a mass distribution, that is a completely additive function of Borel set in $E^{n}$ and suppose that the total variation $V$ of $\mu(P)$ in $E^{n}$ is finite. Then the integral

$$
\tilde{f}_{\lambda}(P)=\int_{E^{n}} K_{\lambda}(P-Q) d \mu(Q)
$$

has a limit $\tilde{f}$ almost everywhere as $\lambda$ tends to infinity, and over every set $S$ of finite measure we have

$$
\int_{S}|\tilde{f}|^{1-\varepsilon} d P \leq \frac{c}{\varepsilon}|S|^{\varepsilon} V^{1-\varepsilon}
$$


Proof. We may assume that $\mu(P) \geq 0$, and we shall show that, given any sphere of finite radius and an $\varepsilon>0$, the integral converges in that sphere outside a subset of measure less than $\varepsilon$. We shall begin with the following observation. Let $P$ be a point, $I_{\varrho}$ a cube with center at $P$ and edge equal to $\varrho$, and $D$ any set contained in $I_{\varrho}$ such that $|D| \geq \alpha\left|I_{\varrho}\right|, \alpha$ being a fixed positive number. Then

exists and

$$
\lim _{\varrho \rightarrow 0} \frac{\mu(D)}{|D|}
$$

is finite for almost every $P$ in $E^{n}$.

$$
\sup _{e} \frac{\mu(D)}{|D|}
$$

Let now $S$ be an arbitrary sphere and $\bar{S}$ the sphere with the same center as $S$ and radius twice as large. Fix $\alpha=2^{-2 n}$. Given an $\varepsilon>0$ choose $y$ so large that the set of points $P$ in $\bar{S}$ such that

$$
\sup _{\varrho} \frac{\mu(D)}{|D|}>y
$$

be of measure less than $\varepsilon 2^{-n} n^{-n / 2}$, and let $A$ be an open set covering this set and the set of measure zero carrying the singular part of $\mu$ in $\bar{S}$, and such that also $|A| \leq \varepsilon 2^{-n} n^{-n / 2}$. Now call $x_{1}, x_{2}, \ldots, x_{n}$ the coordinates of a point in $E^{n}$ and cover $A$ by means of half open cubes

$$
\frac{m_{i}}{2^{k}} \leq x_{i}<\frac{m_{i}+1}{2^{k}} ; \quad(i=1,2, \ldots, n)
$$

where $m_{i}$ and $k$ are integers, in the following manner: first let $k_{0}$ be the smallest value of $k$ for which there is a cube of the above form entirely contained in $A$, and take all such cubes contained in $A_{\text {; }}$ then let $k=k_{0}+1$ and take all the new cubes contained in the remaining part of $A$ and so on. Thus we shall obtain $A$ as the union of non-overlapping half-open eubes which we shall denote by $I_{k}$ with the property that every $I_{k}$ is contained in a cube with edge twice as long and containing a point outside $A$.

Denote by $A_{1}$ the union of all those $I_{k}$ intersecting $S$. Then it is clear that, if $\varepsilon$ is sufficiently small, every $I_{k}$ in $A_{1}$ will be contained in a cube with edge twice as long and containing a point $P$ outside $A$ and in $\bar{S}$. Thus from the definitions of $A$ and of $\alpha$ it follows that $\mu\left(I_{k}\right) \leq y\left|I_{k}\right|$ for every $I_{k} \in A_{1}$. Moreover, outside $A$ and in $\bar{S}$, and therefore also outside $A_{1}$ and in $S$, the function $\mu(P)$ is absolutely continuous and its derivative does not exceed $y$. Let finally $A_{2}$ be the union of all 
spheres $S_{k}$ with center at the centers of $I_{k}$ and radius equal to the diameter of $I_{k} \subset A_{1}$. Clearly, $\left|A_{2}\right| \leq n^{n / 2} 2^{n}\left|A_{1}\right|<\varepsilon$. We shall show that outside $A_{2}$ and in $S$ the integral converges almost everywhere. For this purpose let $g(P)$ be the function equal to the derivative of $\mu(P)$ outside $A_{1}$ and in $S$, equal to $\mu\left(I_{k}\right)\left|I_{k}\right|^{-1}$ in every cube $I_{k}$ of $A_{1}$, and equal to zero elsewhere. Let also $\nu(P)$ be equal to $\mu(P)$ minus the indefinite integral of $g(P)$. Then $\nu\left(I_{k}\right)=0, g(P)$ is less than or equal to $y$, and

$$
\int_{I_{k}}|d \nu(P)| \leq 2 y\left|I_{k}\right| \text {. }
$$

Let now $P$ be a point interior to $S$, outside $A_{2}$, and where the density of $A_{1}$ is zero. Then, denoting by a prime the complement of a set,

$$
\begin{aligned}
\int_{E^{n}} K_{\lambda}(P-Q) d \mu(Q) & =\int_{\left(A_{1} \cup S^{\prime}\right.} K_{\lambda}(P-Q) d \mu(Q)+ \\
& \quad+\int_{E^{n}} K_{\lambda}(P-Q) g(Q) d Q+\int_{A_{1}} K_{\lambda}(P-Q) d \nu(Q) .
\end{aligned}
$$

Since $P$ is interior to $S$, its distance to the set $\left(A_{1} \cup S\right)^{\prime}$ is positive and therefore the first integral converges. Moreover since $g(P)$ is a bounded function which vanishes outside a bounded set the second integral converges almost everywhere, so that the whole problem reduces to showing the convergence of the last integral.

We have

$$
\int_{A_{1}} K_{\lambda}(P-Q) \iota \nu(Q)=\sum_{1} \int_{I_{k}} K_{\lambda}(P-Q) d \nu(Q)+\sum_{2} \int_{I_{k}} K_{\lambda}(P-Q) d \nu(Q),
$$

where the first sum is extended over the cubes of $A_{1}$ intersecting the sphere with center at $P$ and radius $1 / \lambda$ and the second over those entirely outside this sphere. Now, since $P$ is outside $A_{2}$, if $I_{k}$ intersects the sphere with center at $P$ and radius $1 / \lambda$ it follows that $I_{k}$ is contained in a sphere with center at $P$ and radius equal to $3 / \lambda$. Therefore we have

$$
\left|\sum_{1} \int_{I_{k}} K_{\lambda}(P-Q) d \nu(Q)\right| \leq \lambda^{n} c \sum_{I_{I_{k}}}|d \nu(Q)| \leq 2 y \lambda^{n} c \sum_{\mathbf{1}}\left|I_{I_{k}}\right| .
$$

But since all the cubes in $\Sigma_{1}$ are contained in the sphere of radius $3 / \lambda$ and $P$ is a point of density zero of $A_{1}$, we have

and therefore

$$
\lim _{\lambda \rightarrow \infty} \lambda^{n} \sum_{1}\left|I_{k}\right|=0
$$

$$
\Sigma_{1} \int_{I_{k}} K_{\lambda}(P-Q) d \nu(Q) \rightarrow 0
$$

as $\lambda$ tends to infinity. 
On the other hand, since $v\left(I_{k}\right)=0$, for every $I_{k}$ in $\Sigma_{\mathbf{2}}$ we have

$$
\int_{I_{k}} K_{\lambda}(P-Q) d \nu(Q)=\int_{I_{k}}\left[K(P-Q)-K\left(P-Q_{k}\right)\right] d \nu(Q),
$$

where $Q_{k}$ is the center of $I_{k}$. Now, on account of the conditions satisfied by $\Omega$ and the definition of $A_{2}$ it follows, as in Lemma 2 of the preceding chapter, that for every $Q$ in $I_{k}$ and $P$ in $A_{2}^{\prime}$ we have

$$
\left|K(P-Q)-K\left(P-Q_{k}\right)\right| \leq c\left|P-Q_{k}\right|^{-n} \omega\left[c\left|I_{k}\right|^{1 / n}\left|P-Q_{k}\right|^{-1}\right]
$$

and therefore

$$
\int_{A^{\prime}{ }_{2}}\left|K(P-Q)-K\left(P-Q_{k}\right)\right| d P \leq \int_{A_{2}^{\prime}} c\left|P-Q_{k}\right|^{-n} \omega\left[c\left|I_{k}\right|^{1 / n}\left|P-Q_{k}\right|^{-1}\right] d P \leq c
$$

$c$ being a constant. Hence

$$
\begin{gathered}
\int_{A^{\prime}{ }_{2}} d P \sum \int_{I_{k}}\left|K(P-Q)-K\left(P-Q_{k}\right)\right||d v(Q)| \leq c \sum \int_{I_{k}}|d \nu(Q)| \leq \\
\leq 2 c y \sum\left|I_{k}\right|=2 c y\left|A_{1}\right|
\end{gathered}
$$

the sum being extended over all intervals in $A_{1}$. But this implies that for almost every $P$ in $A_{2}^{\prime}$ we have

$$
\sum_{2}\left|\int_{I_{k}} K_{\lambda}(P-Q) d v(Q)\right| \leq \sum \int_{I_{k}}\left|K(P-Q)-K\left(P-Q_{k}\right)\right||d \nu(Q)|<\infty,
$$

and since each of the terms on the left hand side converges as $\lambda \rightarrow \infty$ and is majorized by the corresponding term on the right which is independent of $\lambda$, it follows that

$$
\sum_{2} \int_{I_{k}} K_{\lambda}(P-Q) d v(Q)
$$

converges as $\lambda \rightarrow \infty$.

Thus we have proved that $\tilde{f}_{\lambda}(F)$ converges almost everywhere to a finite limit.

Finally, the last part of the theorem is an immediate consequence of Theorem 4 of Chapter I and Fatou's lemma.

Remark. Suppose that the mass distribution is differentiable at the point $P$. Denoting by $\mu^{\prime}(P)$ the value of the derivative (of course, $\mu^{\prime}(P)$ is a real-valued function of the point) let us consider the mass distribution $\mu_{P}(Q)=\mu(Q)-\mu^{\prime}(P) \chi(Q)$, where $\chi(Q)$ is the indefinite integral of 1 . Due to the properties of the kernel $K$, the two integrals

$$
\int_{E_{n}} K(P-Q) d \mu(Q), \quad \int_{E_{n}} K(P-Q) d \mu_{P}(Q)
$$


converge or diverge simultaneously, and in the case of convergence their values are the same. Let $\Gamma_{\varrho}(P)$ denote the sphere with center $P$ and radius $\varrho$. At almost every point $P$ we have

$$
\int_{\Gamma^{(P)}}\left|d \mu_{P}(Q)\right|=o\left(\varrho^{n}\right) \quad(\varrho \rightarrow 0)
$$

(an analogue of Lebesgue's condition). At every point $P$ at which this condition holds, the convergence of the second integral is to a great extent independent of the shape of the neighborhood excluded around $P$. For let $D_{\varepsilon}(P)$ be any set containing $\Gamma_{\varepsilon}(P)$ and contained in $\Gamma_{M \varepsilon}(P), M$ being a fixed number. Then the difference between the integrals

$$
\int_{D_{\varepsilon}^{\prime}(P)} K(P-Q) d \mu_{P}(Q), \int_{\Gamma_{\varepsilon}^{\prime}(P)} K(P-Q) d \mu_{P}(Q)
$$

(where $\Gamma^{\prime \prime}$ and $D^{\prime}$ are the complements of $\Gamma$ and $D$ ) is numerically

$$
\underset{\Gamma_{M \varepsilon}(P)}{\leq} \int_{\Gamma_{\varepsilon}(P)}|K(P-Q)|\left|d \mu_{P}(Q)\right|=O\left(\varepsilon^{-n}\right) o(M \varepsilon)^{n}=o(1)
$$

and so tends to 0 with $\varepsilon$. Thus, for almost every $P$, and for the sets $D_{\varepsilon}$ of the type just described (we might call them regular neighborhoods) we have

$$
\tilde{f}(P)=\lim _{\varepsilon \rightarrow 0}\left\{\int_{D_{\varepsilon}^{\prime}(P)} K(P-Q) d \mu(Q)+\mu^{\prime}(P) \int_{D_{\varepsilon^{\prime}}^{\prime}(P)} K(P-Q) d Q\right\}
$$

almost everywhere. The second integral on the right here exists for every $\varepsilon>0$. If it tends to 0 with $\varepsilon$ (a situation which can oceur, due to possible symmetries in the structures of $K$ and of $D$ ), then in the last formula we may drop the second term on the right. This is, for example, the case of the kernels (3) and (5) of Introduction, if $D_{\varepsilon}(P)$ is any square with center $P$ and sides $2 \varepsilon$.

\section{CHAPTER III.}

The preceding results can be used to establish differential properties of certain functions. We shall primarily consider the problems of the existence of the first derivatives of the Newtonian potential of a single layer, and of the second derivatives of the logarithmic potential (more general situations we shall consider elsewhere). Thus again we shall be concerned with the kernels of the forms

$$
\frac{x}{\left(x^{2}+y^{2}\right)^{3 / 2}}, \quad \frac{x^{2}-y^{2}}{\left(x^{2}+y^{2}\right)^{2}}, \quad \frac{x y}{\left(x^{2}+y^{2}\right)^{2}} .
$$

Let us consider a mass distribution $\mu$ over the plane, and the Newtonian potential 


$$
u(x, y, z)=\iint_{E^{2}} \frac{1}{R} d \mu(s, t), \quad R=\left[(x-s)^{2}+(y-t)^{2}+z^{2}\right]^{1 / 2}
$$

in the half-space $z>0$. We assume that the total mass $\int|d \mu|$ is finite. Obviously

$$
u_{x}(x, y, z)=\iint_{E^{3}} \frac{s-x}{R^{3}} d \mu(s, t) .
$$

Suppose that the point $\left(x_{0}, y_{0}, z\right)$ approaches $\left(x_{0}, y_{0}, 0\right)$ vertically, and that the mass $\mu$ has a density at $\left(x_{0}, y_{0}\right)$ (by this we mean that $\mu$ is differentiable at $\left(x_{0}, y_{0}\right)$, with respect, say, to concentric circles). Without loss of generality we may assume that $\left(x_{0}, y_{0}\right)=(0,0)$ and that the density in question is zero. Let us split the last integral into two, $P$ and $Q$, the former extended over the circle $\Gamma_{z}$ defined by the equation $s^{2}+t^{2} \leq z^{2}$, and the latter over the complement $\Gamma_{z}^{\prime}$ of $\Gamma_{z}$. If we set

$$
I(r)=\iint_{\Gamma_{\tau}} s d \mu(s, t)
$$

then, as easily seen, $I(r)=o\left(r^{3}\right)$ for $r \rightarrow 0$. Hence

$$
\begin{aligned}
P & =\int_{0}^{z} \frac{d I(r)}{\left(r^{2}+z^{2}\right)^{3 / 2}}=\frac{I(z)}{\left(2 z^{2}\right)^{3 / 2}}+3 \int_{0}^{z} \frac{r I(r) d r}{\left(r^{2}+z^{2}\right)^{5 / 2}}=o(1)+z^{-5} \int_{0}^{z} o\left(r^{4}\right) d r=o(1), \\
Q & -\int_{\Gamma_{z}^{\prime}} \frac{s d \mu}{\left(s^{2}+t^{2}\right)^{3 / 2}}=\int_{z}^{\infty}\left[\frac{1}{\left(r^{2}+z^{2}\right)^{3 / 2}}-\frac{1}{\left(r^{2}\right)^{3 / 2}}\right] d I(r)= \\
& =I(z)\left[\frac{1}{\left(2 z^{2}\right)^{3 / 2}}-\frac{1}{\left(z^{2}\right)^{3 / 2}}\right]+3 \int_{z}^{\infty} r I(r)\left[\frac{1}{\left(r^{2}+z^{2}\right)^{5 / 2}}-\frac{1}{r^{5}}\right] d r= \\
& =o\left(z^{3}\right) O\left(z^{-3}\right)+3 \int_{\dot{z}}^{\infty} o\left(r^{4}\right) O\left(\frac{z^{2}}{r^{4}}\right) d r= \\
& =o(1)+z^{2} \int_{z}^{\infty} o\left(r^{-3}\right) d r=o(1) .
\end{aligned}
$$

Collecting the results, we see that at every point of differentiability of $\mu$ the difference

$$
u_{x}(x, y, z)-\int_{\Gamma^{\prime}} \int_{z} \frac{x-s}{\left[(x-s)^{2}+(y-t)^{2}\right]^{3 / 2}} d \mu(s, t)
$$


converges to 0 as $z \rightarrow+0$. In particular, since the integral

$$
\iint \frac{x-s}{\left[(x-s)^{2}+(y-t)^{2}\right]^{3 / 2}} d \mu(s, t)
$$

exists almost everywhere, we see that $\lim u_{x}(x, y, z)$ exists for almost every $(x, y)$ as $z \rightarrow+0$. A slight - and well known - modification of the above argument shows that $\lim u_{x}(x, y, z)$ exists for almost every point $(x, y)$ as $(x, y, z)$ approaches $(x, y, 0)$ non-tangentially.

Let us now assume that $\mu$ is absolutely continuous, that is that $d \mu=f d s d t$, with $f$ integrable. We shall investigate the problem of the differentiability of the function

$$
u(x, y)=\iint_{E^{2}} \frac{f(s, t) d s d t}{\left[(x-s)^{2}+(y-t)^{2}\right]^{1 / 2}}
$$

which is the potential $u$ in the plane $z=0$.

In what follows, we shall systematically denote by $\Gamma_{r}(x, y)$ the circle with center $(x, y)$ and radius $r$. The complementary set will be denoted by $\Gamma_{r}^{\prime}(x, y)$. Instead of $\Gamma_{r}(0,0)$ and $\Gamma_{r}^{\prime}(0,0)$ we shall simply write $\Gamma_{r}$ and $\Gamma_{r}^{\prime}$.

\section{Theorem 1.}

a) Suppose that $f$ is integrable over the whole plane and that $|f| \log ^{+}|f|$ is integrable over every finite circle of the plane. Then the integral (2) converges over almost every line parallel to the $x$-axis and represents an absolutely continuous ${ }^{1}$ function of $x$. In particular, $u_{x}$ exists almost everywhere. Moreover

almost everywhere.

$$
u_{x}(x, y)=\iint_{E^{2}} \frac{(s-x) f(s, t)}{\left[(x-s)^{2}+(y-t)^{2}\right]^{3 / 2}} d s d t
$$

b) If $f$ is integrable over the whole plane and belongs to $L^{q}, q>2$, over every finite circle, then $u(x, y)$ has a complete differential at almost every point of the plane.

Proof. It slightly simplifies the argument (though it is not essential for the proof), if we assume that $f$ vanishes outside a sufficiently large circle.

Let us consider

$$
u^{(\varepsilon)}(x, y)=\iint_{E^{2}} f(x-s, y-t) \frac{1}{\left(s^{2}+t^{2}+\varepsilon^{2}\right)^{1 / 2}} d s d t
$$

\footnotetext{
1 i.e. absolutely continuous over every finite interval.
} 
and let us compare

with the function

$$
u_{x}^{(\varepsilon)}(x, y)=-\iint_{E^{2}} f(x-s, y-t) \frac{s}{\left(s^{2}+t^{2}+\varepsilon^{2}\right)^{3 / 2}} d s d t
$$

We have

$$
\tilde{f}_{\varepsilon}(x, y)=-\int_{\Gamma_{\varepsilon}^{\prime}} \int_{\varepsilon} f(x-s, y-t) \frac{s}{\left(s^{2}+t^{2}\right)^{3 / 2}} d s d t .
$$

$$
\begin{aligned}
-u_{x}^{(\varepsilon)}(x, y) & +\tilde{f}_{\varepsilon}(x, y)=\iint_{\Gamma_{\varepsilon}} f(x-s, y-t) \frac{s}{\left(s^{2}+t^{2}+\varepsilon^{2}\right)^{3 / 2}} d s d t+ \\
& +\iint_{\Gamma_{\varepsilon}^{\prime}} f(x-s, y-t)\left[\frac{s}{\left(s^{2}+t^{2}+\varepsilon^{2}\right)^{3 / 2}}-\frac{s}{\left(s^{2}+t^{2}\right)^{3 / 2}}\right] d s d t,
\end{aligned}
$$

and if we set

it is readily seen that

$$
N(x, y)=\left\{\begin{array}{l}
\frac{x}{\left(x^{2}+y^{2}+1\right)^{3 / 2}} \text { for } x^{2}+y^{2} \leq 1 \\
\frac{x}{\left(x^{2}+y^{2}+1\right)^{3 / 2}}-\frac{x}{\left(x^{2}+y^{2}\right)^{3 / 2}} \text { for } x^{2}+y^{2}>1
\end{array}\right.
$$

$$
-u_{x}^{(\varepsilon)}(x, y)+\tilde{f}_{\varepsilon}(x, y)=\frac{1}{\varepsilon^{2}} \int_{E^{\mathbf{q}}} \int f(s, t) N\left(\frac{x-s}{\varepsilon}, \frac{y-t}{\varepsilon}\right) d s d t
$$

Now, an application of Lemma 2 in Chapter II shows that, as $\varepsilon \rightarrow 0,-u_{x}^{(\varepsilon)}(x, y)+$ $+\tilde{f}_{\varepsilon}(x, y)$ converges to zero in the mean of order 1 , and, according to Theorem 7 of Chapter I, over every set of finite measure $\tilde{f}_{\varepsilon}(x, y)$ converges in the mean to the function

$$
\tilde{f}(x, y)=-\iint_{E^{2}} f(x-s, y-t) \frac{s}{\left(s^{2}+t^{2}\right)^{3 / 2}} d s d t
$$

Thus, over every set of finite measure, $u_{c}^{(s)}(x, y)$ converges in the mean to $\tilde{f}(x, y)$.

Now we can select a sequence $\varepsilon_{n} \rightarrow 0$ such that, for almost every line $y=y_{0}$, $u_{x}^{(\varepsilon)}\left(x, y_{0}\right)$ converge in the mean to $\tilde{f}\left(x, y_{0}\right)$ over sets of finite measure, and thus

1 If $f_{n}(x, y)$ converges in the mean to $f(x, y)$, we have

$$
\int d y \int\left|f_{n}(x, y) \rightarrow f(x, y)\right| d x \rightarrow 0 .
$$

Thus the inner integral, as a function of $y$, converges in the mean to zero, and we can select a sequence $n_{i}$ such that

$$
\int\left|f_{n_{i}}(x, y)-f(x, y)\right| d x \rightarrow 0
$$

for almost every $y$. 


$$
\lim _{n \rightarrow \infty}\left[u^{\left(\varepsilon_{n}\right)}\left(x, y_{0}\right)-u^{\left(\varepsilon_{n}\right)}\left(x_{0}, y_{0}\right)\right]=\int_{\dot{x}_{0}}^{x} \tilde{f}\left(s, y_{0}\right) d s .
$$

If (as we may assume) $f \geq 0$, the integrand in (3) increases as $\varepsilon$ decreases, and this implies that

$$
\lim _{\varepsilon \rightarrow 0} u^{(\varepsilon)}(x, y)=\iint_{E^{2}} f(x-s, y-t) \frac{1}{\left(s^{2}+t^{2}\right)^{1 / 2}} d s d t=u(x, y) .
$$

Combining the two last results, part a) follows.

Part b) of Theorem 1 asserts that in the neighborhood of almost every point $\left(x_{0}, y_{0}\right)$, the difference $u\left(x_{0}+h, y_{0}+k\right)-u\left(x_{0}, y_{0}\right)$ is of the form $A h+B k+$ $+o\left(h^{2}+k^{2}\right)^{1 / 2}$, where $A=A\left(x_{0}, y_{0}\right), B=B\left(x_{0}, y_{0}\right)$. This is more than the mere existence of the partial derivatives $u_{x}\left(x_{0} y_{0}\right)$ and $u_{y}\left(x_{0}, y_{0}\right)$ established in a), and it implies, in particular, that $u$ is bounded in the neighborhood of $\left(x_{0}, y_{0}\right)$. The mere boundedness of $u$, however, over every finite circle is a direct consequence of Hölder's inequality applied to the integral in (2), since the kernel $\left(s^{2}+t^{2}\right)^{-1 / 2}$ belongs to $L^{p}, p<2$, over every finite circle.

Let us now consider any point $\left(x_{0}, y_{0}\right)$ at which the integral of $\left|f(x, y)-f\left(x_{0}, y_{0}\right)\right|^{q}$ is differentiable and the derivative is zero. (Generalized Lebesgue condition _- it implies ordinary Lebesgue condition with $q=1$ ). Let us also assume that both integrals

$$
\begin{aligned}
& A=-\iint_{E^{2}} f(x-s, y-t) \frac{s}{\left(s^{2}+t^{2}\right)^{3 / 2}} d s d t, \\
& B=-\iint_{E^{2}} f(x-s, y-t) \frac{t}{\left(s^{2}+t^{2}\right)^{3 / 2}} d s d t .
\end{aligned}
$$

exist. Let $\left(h^{2}+k^{2}\right)^{1 / 2}=\varepsilon$, and let us split the integral defining the difference $\Delta=u\left(x_{0}+h, y_{0}+k\right)-u\left(x_{0}: y_{0}\right)$ into two, extended respectively over the circle $\Gamma_{2 \varepsilon}\left(x_{0}, y_{0}\right)$ and its complement $\Gamma_{2 \varepsilon}^{\prime}\left(x_{0}, y_{0}\right)$. Let us denote the integrals so obtained by $P$ and $Q$ respectively. Without loss of generality we may assume that $\left(x_{0}, y_{0}\right)=$ $(0,0)$ and that $f\left(x_{0}, y_{0}\right)=0$. Then, with $H(x, y)=\left(x^{2}+y^{2}\right)^{-1 / 2}$, we have

$$
\begin{aligned}
Q & =\iint_{\Gamma^{\prime}{ }_{2 \varepsilon}} f(s, t)[H(s-h, t-k)-H(s, t)] d s d t=h A_{2 \varepsilon}+k B_{2 \varepsilon}+ \\
& +\frac{1}{2} \iint_{\Gamma_{2 \varepsilon}^{\prime}} f(s, t)\left[h^{2} H_{x x}(s-\theta h, t-\theta k)+2 k h H_{x y}(s-\theta h, t-\theta k)+\right. \\
& \left.+k^{2} H_{y y}(s-\theta h, t-\theta k)\right] d s d t
\end{aligned}
$$


where $\theta$ is a function of $s$ and $t$ such that $0<\theta<1, A_{2 \varepsilon}$ and $B_{2 \varepsilon}$ are the integrals (4) extended over $\Gamma_{2 \varepsilon}^{\prime}$.

If we replace here $A_{2 \varepsilon}$ and $B_{2 \varepsilon}$ by $A$ and $B$, we ultimately commit an error $o(|h|)+o(|k|)=o\left(h^{2}+k^{2}\right)^{1 / 2}$. The first of the remaining three integrals is numerically

$$
\begin{aligned}
O\left(h^{2}\right) \iint_{\Gamma^{\prime} \varepsilon \varepsilon}|f(s, t)|\left[(s-\theta h)^{2}+(t-\theta k)^{2}\right]^{-3 / 2} d s d t & = \\
& =O\left(\varepsilon^{2}\right) \iint_{\Gamma^{\prime} 2 \varepsilon}|f(s, t)|\left(s^{2}+t^{2}\right)^{-3 / 2} d s d t=O\left(\varepsilon^{2}\right) \int_{23}^{\infty} \frac{d I(r)}{r^{3}},
\end{aligned}
$$

where $I(r)$ is the integral of $|f|$ extended over the circle $\Gamma_{r}$. Integration by parts and the fact that, by assumption, $I(r)=o\left(r^{2}\right)$, shows that the last expression is $O\left(\varepsilon^{2}\right) \circ\left(\varepsilon^{-1}\right)=o(\varepsilon)$.

The same remark applies to the remaining two integrals constituting $Q$. Hence

$$
Q=A h+B k+o(\varepsilon) \text {. }
$$

An application of Hölder's inequality to the integrals defining $P$ gives, with $p=\frac{q-1}{q}$.

$$
\begin{gathered}
|P| \leq\left[\iint_{\Gamma_{2 \varepsilon}}|f|^{q} d s d t\right]^{1 / q}\left\{\left[\iint_{\Gamma_{2 \varepsilon}} H^{p} d s d t\right]^{1 / p}+\left[\iint_{\Gamma_{2 \varepsilon}} H(s-h, t-k)^{p} d s d t\right]^{1 / p}\right\} \leq \\
\leq 2\left[\iint_{\Gamma_{2 \varepsilon}}|f|^{\alpha} d s d t\right]^{1 / q}\left[\iint_{\Gamma_{4 \varepsilon}} H^{p} d s d t\right]^{1 / p}=o(\varepsilon) .
\end{gathered}
$$

Hence, using (5), we get $\Delta=P+Q=A h+B k+o(\varepsilon)$, and part b) of Theorem 1 is established.

Remarks. Neither part of Theorem 1 admits of much improvement. For, beginning with part a), let us assume that $f(s, t)$ vanishes outside the square $S, 0 \leq s \leq 1,0 \leq t \leq 1$, and that it is constant, equal to $\varphi\left(s_{0}\right)$, along every segment $s=s_{0}, 0 \leq t \leq 1$. Then, integrating with respect to $t$, one finds that in every smaller square concentric with, and situated. similarly to, $S$ the function $u(x, y)$ differs from the logarithmic potential

$$
L(x, y)=L(x)=\int_{0}^{1} \varphi(s) \log |x-s|^{-1} d s
$$

by a bounded function. Let us suppose that $\varphi$ is non-negative. If $\omega(x)$ is any function tending to $\infty$ with $x$, then the integrability of $\omega(f)$ over $S$ is equivalent to the integrability of $\omega(\varphi)$ over $0 \leq s \leq 1$. If $\omega(x)=x \log ^{+} x$, then an application 
of Young's inequality (see [7], p. 64) to the integral shows that the integrability of $\varphi \log ^{+} \varphi$ implies the boundedness of $L(x, y)$ over $S$. Suppose, however, that $\omega(x)$ tends to $+\infty$ more slowly than $x \log x$. We can then find a positive function $\varphi(s)$, $0 \leq s \leq 1$, with $\omega[\varphi(s)]$ integrable and such that the integral (6) diverges to $+\infty$ in a set of points $x$ dense in $(0,1)$. (See [7], p. 99). Hence $u(x, y)$ equals $+\infty$ on a set of segments $s=s_{0}, 0 \leq t \leq 1$, dense in $S$. This shows that at no point interior to $S$ can $u(x, y)$ have a directional derivative in the direction making an angle of $\pm \frac{\pi}{4}$ with the $x$-axis. Rotating the whole picture by $\frac{\pi}{4}$ we obtain a mass distribution with density $f(s, t)$ such that $\omega(f)$ is integrable and yet the potential $u(x, y)$ has no partial derivative $u_{x}$ or $u_{y}$ at any point interior to a square $S^{\prime}$ obtained by the rotation of $S$.

It is easily seen that $u_{x}$ and $u_{y}$ will be non-existent at almost every point of $S^{\prime}$, no matter how we modify $u(x, y)$ in any set of measure 0 . For, $\varphi$ being $\geq 0$, the function $L(x)$ is lower semicontinuous. Hence given any number $M>0$, no matter how large, we shall have $L(x)=L(x, y)>M$ in a set of strips parallel to the $y$-axis $(\varepsilon \leq y \leq 1-\varepsilon)$ and dense in $0 \leq x \leq 1$. Thus no matter how we modify $u$ in a set of measure 0 it will be discontinuous, in the direction $\pm \frac{\pi}{4}$, at almost every point $(x, y) \in S$.

That in part b) we cannot replace the integrability of $|f|^{q}, q>2$ by the integrability of $f^{2}$ (over every finite circle) is even simpler. For the kernel $H(x, y)=$ $=\left(x^{2}+y^{2}\right)^{1 / 2}$ is not quadratically integrable near the origin. We can therefore construct a function $f(s, t) \geq 0$ quadratically integrable over every finite circle and such that the convolution $u$ of $f$ and $H$ diverges to $+\infty$ in a set dense over the whole plane. It follows that $u$ remains unbounded in every circle no matter how we change $u$ in a set of measure 0 . Thus $u$ cannot have a complete differential at any point, even if we modify $u$ in a set of measure 0 .

Of course, we could slightly sharpen part b) by introducing the logarithmic scale of integrability, but this generalization would be of little interest.

It may also be added that, under the assumptions of Theorem 1, the function $u(x, y)$ is absolutely continuous in Tonelli's sense over every finite square $I$ with sides parallel to the axes. This follows from the fact that $u$ is absolutely continuous on almost every line parallel to one of the axes, and that both $u_{x}$ and $u_{y}$ are integrable over $I$.

On account of certain applications we shall state the analogue of Theorem 1 in $n$ dimensions. 
Theorem 2. Suppose that $f\left(x_{1}, x_{2}, \ldots, x_{n}\right)$ is integrable over the whole space $E^{n}$, and $|f| \log ^{+}|f|$ is integrable over every finite sphere in the space. Then the potential

$$
u\left(x_{1}, \ldots x_{n}\right)=u(P)=\int_{E^{n}}|P-Q|^{-n+1} f(Q) d Q
$$

converges over almost every line $x_{i}=$ const, $i=2, \ldots, n$ and represents an absolutely continuous function of $x_{1}$. In particular the partial derivative $\frac{\partial u}{\partial x_{1}}=u_{x_{1}}$ exists almost everywhere. Moreover

$$
u_{x_{1}}=(n-1) \int_{E^{n}}\left(s_{1}-x_{1}\right)|P-Q|^{-n-1} f(Q) d Q
$$

almost everywhere, $x_{1}$ and $s_{1}$ being the first coordinates of $P$ and $Q$ respectively.

If $f$ is integrable over the whole space $E^{n}$ and belongs to $L^{q}, q>n$, then $u(P)$ has a complete differential at almost every point of the space.

The proof follows very closely that of Theorem 1.

Remark. It is not difficult to see that for the most general mass distribution $d \mu$ the potential $u$ has at almost every point $P=\left(x_{1}, x_{2} \ldots x_{n}\right)$ an approximate differential, that is $u\left(x_{1}+h_{1}, \ldots x_{n}+h_{n}\right)-u\left(x_{1} \ldots x_{n}\right)=\sum_{1}^{n} A_{i} h_{i}+o\left(\sum_{1}^{n}\left|h_{i}\right|\right)$, provided the point $\left(h_{1}, h_{2} \ldots h_{n}\right)$ tends to $(0,0, \ldots 0)$ through a certain set (depending, in general, on $\left.\left(x_{1} x_{2} \ldots x_{n}\right)\right)$ having the origin as a point of strong density. For let us make the usual decomposition $d \mu=g+d \nu$, where $g$ is bounded and coincides with $d \mu$ in a perfect set $S$ and equals the average value of $\mu$ in certain $n$-dimensional cubes constituting the complementary open set $S^{\prime}$. Correspondingly $u=u_{g}+u_{d_{v}}$. Since $g$ is bounded, $u_{g}$ has a differential almost everywhere. Lemma 2 of Chapter I easily shows that $u_{d v}$ has at almost every point a differential with respect to $S$. Making $S$ expand, we obtain the result. This argument shows that $u$ has almost everywhere all the approximate partial derivatives $u_{x_{i}}$.

We now turn to the logarithmic potential

$$
u(x, y)=\iint_{E^{2}} f(x-s, y-t) \log \frac{1}{\left(s^{2}+t^{2}\right)^{1 / 2}} d s d t
$$

Since we are only interested in the differential properties of $u$, we may again assume that $f$ vanishes outside a sufficiently large circle. We shall investigate the existence 9-523804. Acta mathematica. 88. Imprimé le 30 octobre 1952. 
of the derivatives of the first two orders of $u$, and the existence of the second differential of $u$. We shall say that $u$ has a second differential at a point $\left(x_{0}, y_{0}\right)$ if, for $h$ and $k$ tending to 0 ,

(9) $u\left(x_{0}+h, y_{0}+k\right)-u\left(x_{0} y_{0}\right)=A h+B k+\frac{1}{2}\left(C h^{2}+2 D h k+E k^{2}\right)+o\left(h^{2}+k^{2}\right)$

where $A, B, C, D, E$ are independent of $h$ and $k$. The existence of the second differential implies that of the first, and in particular that of $u_{x}\left(x_{0}, y_{0}\right)=A$ and $u_{y}\left(x_{0}, y_{0}\right)=B$. In general, however, it does not imply the existence of the second partial derivatives in the classical sense. For example, for $k=0$ the preceding equation reduces to

$$
u\left(x_{0}+h, y_{0}\right)-u\left(x_{0}, y_{0}\right)=A h+\frac{1}{2} C h^{2}+o\left(h^{2}\right),
$$

which only implies that $u\left(x, y_{0}\right)$ has, for $x=x_{0}$, a second generalized derivative in the sense of Peano and de la Vallée Poussin (see e.g. [7] p. 257).

Theorem 3. Suppose that $f(s, t)$ vanishes outside a circle and that $|f| \log ^{+} \mid f$ is integrable (in particular $f \in L$ ). Then

a) the integral (8) converges absolutely and represents a continuous function $u(x, y)$.

b) On almost every line parallel to either axis, $u(x, y)$ is continuously differentiable and the integrals

$$
-\iint_{E^{2}} f(x-s, y-t) \frac{s}{s^{2}+t^{2}} d s d t ;-\iint_{E^{2}} f(x-s, y-t) \frac{t}{s^{2}+t^{2}} d s d t
$$

obtained by formal differentiation of the integral (8) converge and represent $u_{x}(x, y)$ and $u_{y}(x, y)$ respectively.

c) On almost every line parallel to either axis the derivatives $u_{x}$ and $u_{y}$ are $a b$ solutely continuous functions. In particular, $u_{x x}, u_{y y}, u_{x y}, u_{y x}$ exist almost everywhere. They are given almost everywhere by the formulae

$$
\begin{aligned}
& u_{x x}(x, y)=-\pi f(x, y)+\iint_{E^{2}} f(x-s, y-t) \frac{s^{2}-t^{2}}{\left(s^{2}+t^{2}\right)^{2}} d s d t \\
& u_{y y}(x, y)=-\pi f(x, y)+\iint_{E^{2}} f(x-s, y-t) \frac{t^{2}-s^{2}}{\left(s^{2}+t^{2}\right)^{2}} d s d t \\
& u_{x y}(x, y)=u_{y x}(x, y)=\iint_{E^{2}} f(x-s, y-t) \frac{2 s t}{\left(s^{2}+t^{2}\right)^{2}} d s d t
\end{aligned}
$$

in particular, $u_{x x}+u_{y y}=-2 \pi f$ almost everywhere. 
d) The function $u(x, y)$ is absolutely continuous (i.e. is an integral).

e) The function $u$ has almost everywhere a second differential, with $C, D$ and $E$ in (9) equal to $u_{x x}, u_{x y}$ and $u_{y y}$ respectively.

Proof. That the integral (8) converges uniformly and absolutely follows from the inequality

$$
x y \leq x \log ^{+} x+e^{y-1},
$$

(See [7] p. 64) applied to the product $|f| \cdot \frac{1}{2} \log r$.

Whithout loss of generality we may assume that $f \geq 0$. Let us consider the function

$$
u^{(\varepsilon)}(x, y)=\frac{1}{2} \iint_{E^{2}} f(x-s, y-t) \log \frac{1}{s^{2}+t^{2}+\varepsilon^{2}} d s d t
$$

and let us compare $u_{x x}^{(\varepsilon)}$ with the function

We have

$$
\tilde{f}_{\varepsilon}(x, y)=\iint_{\Gamma_{\varepsilon}^{\prime}} f(x-s, y-t) \frac{s^{2}-t^{2}}{\left(s^{2}+t^{2}\right)^{2}} d s d t .
$$

$$
\begin{gathered}
u_{x x}^{(\varepsilon)}-\tilde{f}_{\varepsilon}=\iint_{\Gamma_{\varepsilon}} f(x-s, y-t) \frac{s^{2}-t^{2}-\varepsilon^{2}}{\left(s^{2}+t^{2}+\varepsilon^{2}\right)^{2}} d s d t+ \\
+\int_{\Gamma_{\varepsilon}^{\prime}} \int_{\varepsilon^{\prime}} f(x-s, y-t)\left[\frac{s^{2}-t^{2}-\varepsilon^{2}}{\left(s^{2}+t^{2}+\varepsilon^{2}\right)^{2}}-\frac{s^{2}-t^{2}}{\left(s^{2}+t^{2}\right)^{2}}\right] d s d t,
\end{gathered}
$$

and if we set

we may write

$$
N(x, y)=\left\{\begin{array}{l}
\frac{x^{2}-y^{2}-1}{\left(x^{2}+y^{2}+1\right)^{2}} \text { for } x^{2}+y^{2} \leq 1, \\
\frac{x^{2}-y^{2}-1}{\left(x^{2}+y^{2}+1\right)^{2}}-\frac{x^{2}-y^{2}}{\left(x^{2}+y^{2}\right)^{2}} \text { for } x^{2}+y^{2}>1,
\end{array}\right.
$$

$$
u_{x x}^{(\varepsilon)}-\tilde{f}_{\varepsilon}=\frac{1}{\varepsilon^{2}} \int_{E^{2}} \int f(s, t) N\left(\frac{x-s}{\varepsilon}, \frac{y-t}{\varepsilon}\right) d s d t
$$

Then, by Lemma 2 in Chapter II it follows that, as $\varepsilon \rightarrow 0, u_{x x}^{(t)}-\tilde{f}_{\varepsilon}$ converges to $-\pi f(x, y)$ in the mean. But according to Theorem 7 in Chapter I, over every set of finite measure $\tilde{f}_{6}(x, y)$ converges in the mean to the function

and thus

$$
\tilde{f}(x, y)=\iint_{E^{2}} f(x-s, y-t) \frac{s^{2}-t^{2}}{\left(s^{2}+t^{2}\right)^{2}} d s d t
$$




$$
\text { l. i. m. } u_{\varepsilon \rightarrow 0}^{(\varepsilon)}=-\pi f(x, y)+\iint_{E^{2}} f(x-s, y-t) \frac{s^{2}-t^{2}}{\left(s^{2}+t^{2}\right)^{2}} d s d t=f_{11}(x y) .
$$

Similarly we get

$$
\begin{gathered}
\operatorname{l.i.m.}_{\varepsilon \rightarrow 0} u_{x y}^{(\varepsilon)}=\iint_{E^{2}} \int f(x-s, y-t) \frac{2 s t}{\left(s^{2}+t^{2}\right)^{2}} d s d t=f_{12}(x y), \\
\lim _{\varepsilon \rightarrow 0} u_{y y}^{(\varepsilon)}=-\pi f(x, y)+\iint_{E^{2}} \int f(x-s, y-t) \frac{t^{2}-s^{2}}{\left(s^{2}+t^{2}\right)^{2}} d s d t=f_{22}(x y) .
\end{gathered}
$$

For the first derivative of $u^{(\varepsilon)}$ we have

$$
\lim _{\varepsilon \rightarrow 0} u_{x}^{(\varepsilon)}(x y)=\lim _{\varepsilon \rightarrow 0}\left\{-\iint_{E^{2}} f(x-s, y-t) \frac{s d s d t}{s^{2}+t^{2}+\varepsilon^{2}}\right\}=-\iint_{E^{2}} f(x-s, y-t) \frac{s d s d t}{s^{2}+t^{2}}
$$

at every point where the integral

$$
\iint_{E^{2}} f f(x-s, y-t) \frac{1}{\left(s^{2}+t^{2}\right)^{1 / 2}} d s d t
$$

is finite. But according to Theorem 1 of this chapter, this is in fact so at all points of almost every line $y=y_{0}$.

Thus for almost every $y$ and every $x$ we have that $u^{(e)}(x, y)$ converges and

$$
\lim _{\varepsilon \rightarrow 0} u_{x}^{(\varepsilon)}=-\iint_{E^{2}} f(x-s, y-t) \frac{s}{s^{2}+t^{2}} d s d t=f_{1}(x, y) .
$$

An analogous result holds for $u_{y}^{(e)}(x, y)$.

Finally for $u^{(\varepsilon)}(x, y)$ itself we have

$$
\lim _{\varepsilon \rightarrow 0} u^{(\varepsilon)}(x, y)=u(x, y)
$$

everywhere, since the integrand in (10) increases as $\varepsilon$ decreases.

We now select a sequence $\varepsilon_{n} \rightarrow 0$ such that, over every set of finite measure of almost every line $y=y_{0}$, the left-hand sides of (11) converge in the mean to the right hand sides. Let $y=y_{0}$ be such a line where in addition (12) is satisfied, and take any point $\left(x_{0}, y_{0}\right)$ on it. Then

$$
\begin{gathered}
u^{\left(\varepsilon_{n}\right)}\left(x, y_{0}\right)=\int_{x_{0}}^{x}(x-s) u_{x x}^{\left(\varepsilon_{n}\right)}\left(s, y_{0}\right) d s+\left(x-x_{0}\right) u_{x}^{\left(\varepsilon_{n}\right)}\left(x_{0}, y_{0}\right)+u^{\left(\varepsilon_{n}\right)}\left(x_{0}, y_{0}\right) \\
u^{\left(\varepsilon_{n}\right)}\left(x, y_{0}\right)=\int_{x_{0}}^{x} u_{x x}^{\left(\varepsilon_{n}\right)}\left(s, y_{0}\right) d s+u^{\left(\varepsilon_{n}\right)}\left(x_{0}, y_{0}\right),
\end{gathered}
$$


and passing to the limit we obtain

$$
\begin{gathered}
u\left(x, y_{0}\right)=\int_{x_{0}}^{x}(x-s) f_{11}\left(s, y_{0}\right) d s+\left(x-x_{0}\right) f_{1}\left(x_{0}, y_{0}\right)+u\left(x_{0}, y_{0}\right) \\
f_{1}\left(x, y_{0}\right)=\int_{x_{0}}^{x} f_{11}\left(s, y_{0}\right) d s+f_{1}\left(x_{0}, y_{0}\right)
\end{gathered}
$$

for all $x$. A similar result holds for almost every line $x=x_{0}$. This proves the absolute continuity of $u_{x}$ and $u_{y}$, and gives the first two formulas (9a).

Let now $\left(x_{0}, y_{0}\right)$ be a point such that $u$ be continuously differentiable on $x=x_{0}$ and on $y=y_{0}$. Then

$$
u^{(\varepsilon)}(x, y)=\int_{x_{0}}^{x} \int_{y_{0}}^{y} u_{x y}^{(\varepsilon)}(s, t) d s d t+u^{(\varepsilon)}\left(x, y_{0}\right)+u^{(\varepsilon)}\left(x_{0}, y\right)-u^{(\varepsilon)}\left(x_{0}, y_{0}\right)
$$

and passing to the limit we have

$$
u(x, y)=\int_{x_{0}}^{x} \int_{y_{0}}^{y} f_{12}(s, t) d s d t+u\left(x, y_{0}\right)+u\left(x_{0}, y\right)-u\left(x_{0}, y_{0}\right) .
$$

But by a theorem of Tonelli and Fubini [3] for almost every $y=y_{0}$ the derivative with respect to $y$ of the double integral above exists for all $x$ and is equal to

$$
\int_{x_{0}}^{x} f_{12}\left(s, y_{0}\right) d s
$$

and thus is an absolutely continuous function of $x$ whose derivative with respect to $x$ is $f_{12}\left(x, y_{0}\right)$. This completes the proof of part $\left.c\right)$.

The last formula also shows that $u(x, y)$ is absolutely continuous.

It remains to prove that $u(x, y)$ has almost everywhere a second differential.

Let $\left(x_{0}, y_{0}\right)$ be a point such that

1) the indefinite integral of $u_{x y}$ is differentiable at $\left(x_{0}, y_{0}\right)$ with respect to regular rectangles and its derivative is $u_{x y}\left(x_{0}, y_{0}\right)$;

2) $u\left(x_{0}+h, y_{0}\right)=u\left(x_{0}, y_{0}\right)+h u_{x}\left(x_{0}, y_{0}\right)+\frac{h^{2}}{2} u_{x x}\left(x_{0}, y_{0}\right)+o\left(h^{2}\right)$;

3) $u\left(x_{0}, y_{0}+k\right)=u\left(x_{0} y_{0}\right)+k u_{y}\left(x_{0} y_{0}\right)+\frac{k^{2}}{2} u_{y y}\left(x_{0} y_{0}\right)+o\left(k^{2}\right)$

Since each of these conditions is fulfilled almost everywhere, they will also be satisfied simultaneously almost everywhere. Now

$$
\begin{aligned}
& u\left(x_{0}+h, y_{0}+k\right)=\int_{0}^{h} \int_{0}^{k} u_{x y}\left(x_{0}+s, y_{0}+t\right) d s d t+u\left(x_{0}+h, y_{0}\right)+ \\
& +u\left(x_{0}, y_{0}+k\right)-u\left(x_{0}, y_{0}\right)
\end{aligned}
$$


A. P. Calderon and A. Zygmund.

and on account of 1) the double integral is equal to $h k u_{x y}\left(x_{0} y_{0}\right)+o\left(h^{2}+k^{2}\right)^{1}$. Thus, taking 2) and 3) into account part e) follows.

We conclude this paper with an extension of Theorem 3 to the potential

$$
u(P)=\int_{E^{n}} f(Q)|P-Q|^{-(n-2)} d Q \quad(n>2)
$$

in $E^{n}$.

Theorem 4. Suppose that $f(P)=f\left(x_{1}, x_{2}, \ldots, x_{n}\right)$ is integrable over $E^{n}$ and that $|f| \log ^{+}|f|$ is integrable over every sphere. Then

a) The integral (13) converges on almost every two-dimensional plane parallel to a fixed plane and represents a continuous, indeed an absolutely continuous, function there.

b) On almost every line parallel to a fixed line, $u(P)$ is continuously differentiable and the derivative is absolutely continuous. On almost every line parallel to any coordinate axis all the derivatives $u_{x_{1}}, u_{x_{2}}, \ldots u_{x_{n}}$ are absolutely continuous and are given by the formulas

$$
u_{x_{i}}(P)=\int_{E^{n}} f(Q) \frac{\partial}{\partial x_{i}}|P-Q|^{-(n-2)} d Q
$$

In particular, all the second derivatives $u_{x_{i} x_{j}}$ exist almost everywhere. They are given almost everywhere by the formulas.

$$
\begin{gathered}
u_{x_{i} x_{i}}=-v_{n} f(P)+\int_{E^{n}} f(Q) \frac{\partial^{2}}{\partial x_{i}^{2}}|P-Q|^{-(n-2)} d Q \\
u_{x_{i} x_{j}}=\int_{E^{n}} f(Q) \frac{\partial^{2}}{\partial x_{i} \partial x_{j}}|P-Q|^{-(n-2)} d Q \quad(i \neq j)
\end{gathered}
$$

$v_{n}$ denoting the volume of the $n$-dimensional unit sphere. In particular $u_{x_{1} x_{1}}+\cdots+$ $+u_{x_{n} x_{n}}=-n v_{n} f$ almost everywhere.

c) If $f \in L^{q}, q>\frac{n}{2}$, then $u$ has a second differential almost everywhere.

Proof. We begin with c). Let $P$ be a point such that

1 Suppose, in fact, that $0<h<k$. If $\frac{k}{2}<h<k$ our assertion is obviously true. On the other hand, if $h<\frac{k}{2}$ the integral is equal to

$$
\int_{0}^{k} \int_{0}^{k} u_{x y} d s d t-\int_{h}^{k} \int_{0}^{k} u_{x y} d s d t=h k u_{x y}\left(x_{0}, y_{0}\right)+o\left(k^{2}\right)+o[k(k-h)] .
$$




$$
\begin{aligned}
& I_{\varrho}=\int_{\Gamma_{Q}}|f(Q)-f(P)|^{Q} d Q=o\left(\varrho^{n}\right) ; \\
& J_{\varrho}=\int_{\Gamma_{\varrho}}|f(Q)-f(P)| d Q=o\left(\varrho^{n}\right)^{1}
\end{aligned}
$$

where $I_{Q}$ denotes a sphere of radius $\varrho$ with center at $P$. Let us suppose in addition that (14) and (15) hold at $P$. Without any loss of generality we may further assume that $f(P)=0$.

Let $\bar{e}$ be an arbitrary unit vector with components $\alpha_{i}$ and consider the expression

$$
u(P+\varrho \bar{e})-u(P)-\varrho\left(\frac{d u}{d \varrho}\right)-\frac{1}{2} \varrho^{2}\left(\frac{d^{2} u}{d \varrho^{2}}\right)=\Delta(\varrho)
$$

where

$$
\frac{d u}{\dot{u} \varrho}=\sum_{i} \frac{\partial u}{\partial x_{i}} \alpha_{i} ; \quad \frac{d^{2} u}{d \varrho^{2}}=\sum_{i j} \frac{\partial^{2} u}{\partial x_{i} \partial x_{j}} \alpha_{i} \alpha_{j}
$$

the partial derivatives being taken at the point $P$.

If we show that $\Delta(\varrho)=o\left(\varrho^{2}\right)$ uniformly in $\bar{e}$, our assertion will be established.

Let us replace in (17) the corresponding integrals. Denoting the complement of $\Gamma_{Q}$ by $\Gamma_{\varrho}^{\prime}$, we have

$$
\begin{gathered}
\Delta(\varrho)=\int_{\Gamma_{2 \varrho}}\left[|P+\varrho \bar{e}-Q|^{-n+2}-|P-Q|^{-n+2}\right] f(Q) d Q- \\
-\varrho \int_{\Gamma_{2 \varrho}} \frac{d}{d \varrho}|P-Q|^{-n+2} f(Q) d Q-\frac{1}{2} \varrho^{2} \int_{\Gamma_{2 \varrho}} \frac{d^{2}}{d \varrho^{2}}|P-Q|^{-n+2} f(Q) d Q+ \\
+\int_{\Gamma_{2 \varrho}}\left[|P+\varrho \bar{e}-Q|^{-n+2}-|P-Q|^{-n+2}-\varrho \frac{d}{d \varrho}|P-Q|^{-n+2}-\right. \\
\left.-\frac{1}{2} \varrho^{2} \frac{d^{2}}{d \varrho^{2}}|P-Q|^{-n+2}\right] f(Q) d Q=A+B+C+D .
\end{gathered}
$$

First, let us remark that on account of our assumption that $f(P)=0$ in each of the preceding integrals we may replace $f(Q)$ by $f(Q)-f(P)$.

Then, by Hölder's inequality, we have

$$
|A| \leq 2\left[\int_{r_{2}}|f(Q)-f(P)|^{Q} d Q\right]^{1 / Q}\left[\int_{r_{3 \ell}}|P-Q|^{(2-n) p} d Q\right]^{1 / \alpha}
$$

and on account of (16) we get $|A|=o\left(\varrho^{2}\right)$.

For $B$ we have, again on account of (16),

1 It is not difficult to show that $I_{Q}=o\left(\varrho^{n}\right)$ implies that $J_{Q}=o\left(\varrho^{n}\right)$. In fact this follows easily by applying Hölder's inequality to $J_{Q}$. 


$$
\begin{gathered}
|B| \leq(n-2) \varrho \int_{0}^{2 \varrho} r^{-n+1} d J_{r}=(n-2) 2^{-n+1} \varrho^{-n+2} J_{2 \varrho}+ \\
\quad+(n-2)(n-1) \varrho \int_{0}^{2 \varrho} \frac{J_{r}}{r^{n}} d r=o\left(\varrho^{2}\right) .
\end{gathered}
$$

The integral in $C$ converges to zero and thus it follows that also $|C|=o\left(\varrho^{2}\right)$.

Finally, it is easily seen that the quantity in square brackets in the integral $D$ does not exceed $c Q^{3}|P-Q|^{-n-1}$. Thus

$$
|D| \leq c \varrho^{3} \int_{2 \varrho}^{\infty} r^{-n-1} d J_{r}=c 2^{-n-1} \varrho^{-n+2} J_{2 \varrho}+c(n+1) \varrho^{3} \int_{2 \varrho}^{\infty} \frac{\dot{J}_{r}}{r^{n+2}} d r=o\left(\varrho^{2}\right),
$$

and c) is established.

The continuity of $u(P)$ in almost every plane parallel to a given plane, under the assumptions of the integrability of $|f| \log ^{+}|f|$, is less trivial here than the continuity of $u(x, y)$ in. Theorem 4. It is enough, however, to sketch the proof. We may take the $x_{1} x_{2}$ plane for the fixed plane.

First we consider the function

$$
u^{(\varepsilon)}(P)=\int\left[|P-Q|^{2}+\varepsilon^{2}\right]^{-\frac{1}{2}(n-2)} f(Q) d Q \quad(f \geq 0)
$$

and prove that $u^{(\varepsilon)}$ converges everywhere to $u(P)$, and that its first and second derivatives converge $n$ the mean of order 1 over every set of finite measure. Then we select a subsequence $u^{\left(\varepsilon_{n}\right)}$ in such a way that the derivatives converge in the mean over every set of finite measure on almost every plane parallel to the $x_{1} x_{2}$ plane, and on almost every line parallel to the $x_{1}$ or $x_{2}$ axis. We may suppose that the $x_{1} x_{2}$ piane is such a plane and the $x_{1}$ and $x_{2}$ axes are such lines. Then

$$
\begin{aligned}
u^{\varepsilon}\left(x_{1}, x_{2}, 0, \ldots, 0\right) & =u^{(\varepsilon)}(0,0, \ldots, 0)+\int_{0}^{x_{1}} \int_{0}^{x_{2}} \frac{\partial^{2} u^{(\varepsilon)}}{\partial x_{1} \partial x_{2}} d x_{1} d x_{2}+ \\
& +\int_{0}^{x_{1}} \frac{\partial u^{(\varepsilon)}}{\partial x_{1}} d x_{1}+\int_{0}^{x_{2}} \frac{\partial u^{(\varepsilon)}}{\partial x_{2}} d x_{2}
\end{aligned}
$$

and, passing to the limit,

$$
\begin{gathered}
u\left(x_{1}, x_{2}, 0, \ldots, 0\right)=u(0,0, \ldots, 0)+\int_{0}^{x_{1}} \int_{0}^{x_{2}} \lim \frac{\partial^{2} u^{\left(\varepsilon_{n}\right)}}{\partial x_{1} \partial x_{2}} d x_{1} d x_{2}+ \\
+\int_{0}^{x_{1}} \lim \frac{\partial u^{\left(\varepsilon_{n}\right)}}{\partial x_{1}} d x_{1}+\int_{0}^{x_{2}} \lim \frac{\partial u^{\left(\varepsilon_{n}\right)}}{\partial x_{2}} d x_{2} .
\end{gathered}
$$


This shows the existence almost everywhere of the derivatives $u_{x_{1}}, u_{x_{2}}, u_{x_{1} x_{2}}$, and the continuity - even absolute continuity - of $u$ in the $x_{1} x_{2}$ plane. The proof of the remaining statements in Theorem 4 is similar.

As in the case of Theorem 2, we may supplement Theorem 4 by the following remark. Let $u(P)$ be the potential of a general mass distribution $d \mu$. Then almost everywhere $u$ has an approximate second differential, in the sense that in the formula (17) the expression $A(\varrho)$ is $o\left(\varrho^{2}\right)$ for almost every $P, \varrho \bar{e}$ tends to 0 through a set of points having 0 as a point of strong density. The proof follows from the same decomposition $d \mu=g+d v$ as in the case of Theorem 2. The argument also shows that the approximate second derivatives $u_{x_{i} x_{j}}$ exists almost everywhere (being defined as the approximate first derivatives of the ordinary first derivatives) and satisfy the equations (15). In particular, the $u_{x_{i} x_{i}}$ satisfy Poisson's equation.

\section{Added in proof, 1. VIII. 52.}

$1^{\circ}$. When this paper was already accepted for publication, Prof. W. J. Trjitzinsky called our attention to an interesting expository article by S. G. Mikhlin, "Singular integral equations", Uspekhi Matematicheskikh Nauk, No 25 (1948), 29-112, which treats topics similar to those discussed in the present paper and describes the earlier work of Giraud, Tricomi and the author himself. However, only functions of the class $L^{2}$ are considered there, and singular integrals are treated in the sense of mean convergence (in the metric $L^{2}$ ). On the other hand, considering vector-functions and matrix-kernels leads the author naturally to the problems of inversion and of the norm preservation of the transform. (For the case $K(z)=1 / z^{2}, f \in L^{2}$, these problems have also been solved in an unpublished work of Prof. A. Beurling.) Combining those results with the theorems of the present paper, one may present the former in a stronger form, as we hope to show elsewhere.

$2^{\circ}$. In Chapters I and II of the present paper we discussed the case of functions $f$ non-periodic and defined over the entire space $E^{n}$. Analogous results can be obtained for periodic functions. We shall limit ourselves here to describing only the general idea. Let $\bar{e}_{1}, \bar{e}_{2}, \ldots, \bar{e}_{n}$ be a system of $n$ independent vectos in $E^{n}$, which for simplicity we assume to be mutually orthogonal and of length $2 \pi$. Let $P_{0}=O$, $P_{1}, P_{2}, \ldots$ be the sequence of terminal points of the vectors $\left(P_{v}-O_{y}\right)=k_{1} \bar{e}_{1}+$ $+k_{2} \bar{e}_{2}+\cdots+k_{n} \bar{e}_{n}$, where the $k_{j}$ are arbitrary integers. The series on the right in the formula

$$
K^{*}(P-O)=K(P-O)+\sum_{p=1}^{\infty}\left\{K\left(P-P_{v}\right)-K\left(O-P_{\nu}\right)\right\}
$$


converges absolutely and uniformly over any finite sphere in $E^{n}$, provided we drop the first few terms. The function $K^{*}$ is periodic, of period $2 \pi$, in each Cartesian coordinate, and the Fourier coefficients of $K^{*}$, taken in the principal value sense, are equal to the corresponding values of Fourier transform of $K$. If

$f(P) \sim \sum c_{k_{1}, k_{n}} \exp i\left(k_{1} x_{1}+\cdots+k_{n} x_{n}\right)$, the function

$$
f^{*}(P)=(2 \pi)^{-n} \int_{R} f(Q) K^{*}(P-Q) d Q
$$

where $R$ stands for the cube $\left|x_{j}\right| \leq \pi, j=1,2, \ldots, n$, plays a role similar to that of the ordinary conjugate function in $E^{1}$. If $f^{*}$ is integrable, its Fourier coefficients are $c_{k_{1}} \ldots k_{n} \hat{K}_{k_{1}} \ldots k_{n}$, where $\hat{K}_{k_{1} \ldots k_{n}}$ denote the Fourier coefficients of $K^{*}$. Familiar results about $f^{*}$ in $E^{1}$ (in which case $K(t)=1 / t, K^{*}(t)=\frac{1}{2} \cot \frac{1}{2} t$ ) are simple consequences of the theorems established in Chapters I and II and are easily extensible to general $n$. The simplest cases for $n=2$ are the kernels $K(z)=z^{k} /|z|^{k+2}, k= \pm 1$, $\pm 2, \ldots$ The kernel $K^{*}$ associated with $K(z)=1 / z^{2}$ is the classical $\wp$ function of Weierstrass.

$3^{\circ}$. Let $\bar{e}_{1}, \bar{e}_{2}, \ldots, \bar{e}_{n}$ be any system of independent vectors in $E^{n}$ and $P_{0}=O$, $P_{1}, P_{2}, \ldots$ the set of lattice points generated by this system. Let $x_{0}, x_{1}, \ldots$ be any sequence of complex numbers such that $\Sigma\left|x_{\mu}\right|^{p}<\infty$, where $p>1$ is a fixed number, and let

$$
\tilde{x}_{\nu}=\sum_{\mu \neq \nu} x_{\mu} K\left(P_{v}-P_{\mu}\right)
$$

Theorem I of Chapter I leads to the inequality

$$
\left(\Sigma\left|\tilde{x}_{v}\right|^{p}\right)^{1 / p} \leq A_{p}\left(\Sigma\left|x_{\mu}\right|^{p}\right)^{1 / p}
$$

(For $n=1$ this remark is due to M. Riesz [9], and the proof in the case of general $n$ follows a similar pattern). The last inequality can be written in the form

$$
\left|\Sigma x_{\mu} y_{v} K\left(P_{v}-P_{\mu}\right)\right| \leq A_{p}\left(\Sigma\left|x_{\mu}\right|^{p}\right)^{1 / p}\left(\Sigma\left|y_{v}\right|^{q}\right)^{1 / q}
$$

where $p>1, q>1,1 / p+1 / q=1$. The case $n=2, K(z)=1 / z^{2}$ is of special interest. The equations $(*)$ can then also be written

$$
\tilde{x}_{\nu}=\sum_{\mu \neq \nu} x_{\mu}(\nu-\mu)^{-2}
$$

where $\mu$ and $\nu$ now run through all complex integers. This may be considered as the simplest generalization of the Hilbert-Toeplitz linear form to space $E^{2}$. The norm of this transformation is the upper bound of the modulus of the function defined by the Fourier series $\Sigma^{\prime}(k+i l)^{-2} \exp i(k x+l y)$. 
$4^{\circ}$. Theorem 1 of Chapter 1 can be written in the form (which again for $n=1$ was pointed out by M. Riesz)

$$
\left|\int_{E^{n}} \int_{E^{n}} f(P) g(Q) K(P-Q) d P d Q\right|=A_{p}\|f\|_{p}\|g\|_{q}(1 / p+1 / q=1),
$$

where the integral on the left is considered as the limit, for $\varepsilon \rightarrow 0$, of the integral extended over the portion $|P-Q| \geq \varepsilon$ of the space $E^{n} \times E^{n}$.

$5^{\circ}$. The case $K(P-O)=(P-O)|P-O|^{-n-1}, f \in L^{2}$ is also discussed in a recent unpublished paper of J. Horváth.

\section{References.}

[1] G. C. Evass, On potentials of positive masses, Transactions of the American Math. Soc., 37 (1935).

[2] K. O. Frredrichs, A theorem of Lichtenstein, Duke Math. Journal, 14, 67--82 (1947).

[3] G. Fubini and L. Tonelli, Sulla derivata seconda mista di un integrale doppio, Rendiconti Circolo Mat. di Palermo, 40, 295-298 (1915).

[4] B. Jessen, J. Marcinkiewicz and A. Zygmund, Note on the differentiability of multiple integrals, Fundamenta Math., 25, 217-234 (1935).

[5] H. Kober, A note on Hilbert transforms, Journal of the London Math. Soc., 18, $66-71$ (1943).

[6] L. Lichtenstein, Über das Poissonsche Integral, Journal für reine und angewandte Mathematik, 141, 12—42 (1912).

[7] A. Zygmund, Trigonometrical Series, Warsaw (1936).

[8] N. Aronszajn, Propriétés de certaines classes hilbertiennes complétées, Comptes rendus de l'Académie des Sciences de Paris, 226, 700-702 (1948).

[9] M. RIEsz, Sur les fonctions conjuguées, Math. Zeitschrift, 27, 218-244 (1927). 